\title{
Advances in Remote Sensing of Post-Fire Vegetation Recovery Monitoring - A Review
}

\author{
Ioannis Gitas¹, George Mitri², \\ Sander Veraverbeke ${ }^{3,4}$ and Anastasia Polychronaki ${ }^{1}$ \\ ${ }^{1}$ Laboratory of Forest Management and Remote Sensing, \\ Aristotle University of Thessaloniki, Thessaloniki, \\ ${ }^{2}$ Biodiversity Program, Institute of the Environment, \\ University of Balamand and Department of Environmental Sciences, \\ Faculty of Science, University of Balamand, \\ ${ }^{3}$ Department of Geography, Ghent University, Ghent \\ ${ }^{4}$ Jet Propulsion Laboratory, California Institute of Technology, Pasadena, CA, \\ ${ }^{1}$ Greece \\ ${ }^{2}$ Lebanon \\ ${ }^{3}$ Belgium \\ ${ }^{4}$ USA
}

\section{Introduction}

Accurate information relating to the impact of fire on the environment and the way it is distributed throughout the burned area is a key factor in quantifying the impact of fires on landscapes (van Wagtendonk et al. 2004), selecting and prioritizing treatments applied on site (Patterson and Yool 1998), planning and monitoring restoration and recovery activities (Jakubauskas 1988; Jakubauskas et al. 1990; Gitas 1999) and, finally, providing baseline information for future monitoring (Brewer et al. 2005).

In order to assess economic losses and ecological effects, post-fire impact assessment requires precise information on extent, type and severity of fire (short-term impact assessment) as well as on forest regeneration and vegetation recovery (long-term impact assessment). Assessing the short-term impact is related to the study of fire behaviour, fire suppression and fire effects while the long-term impact assessment of fires is needed in order to establish post-fire monitoring management and introduce restoration and recovery activities.

As fire sizes increase and time becomes a constraining factor, traditional methods to assess post-fire impact on vegetation have become costly and labour-intensive (Bertolette and Spotskey 2001; Mitri and Gitas 2008). Given the extremely broad spatial expanse and often limited accessibility of the areas affected by fire, satellite remote sensing is an essential technology for gathering post-fire related information in a cost-effective and time-saving manner (Smith and Woodgate 1985; Chuvieco and Congalton 1988; Jakubauskas et al. 1990; White et al. 1996; Patterson and Yool 1998; Beaty and Taylor 2001; Escuin et al. 2002). 
In addition, the development of high spatial and spectral resolution remote sensing instruments, both airborne and spaceborne, as well as advanced image analysis techniques have provided an opportunity to evaluate patterns of forest regeneration and vegetation recovery after wildfire.

The aim of this chapter is to review the role of Remote Sensing (RS) in post-fire monitoring of vegetation recovery. More specifically, traditional and advanced methods and techniques that have been so far employed to monitor vegetation regrowth after fire by RS will be reviewed and future trends will be identified.

More specifically, Part 2 deals with the ecological framework of the effects of fire on the ecosystem, Part 3 describes the methods and techniques that have so far been employed to estimate forest regeneration and vegetation recovery by means of field survey and by RS, Part 4 focuses on the advances in RS of post-fire vegetation monitoring, Part 5 emphasises on future trends in RS of post-fire monitoring, and Part 6 outlines the main conclusions of the chapter.

\section{Ecological framework}

Fire is an integral part of many ecosystems (Trabaud 1994). However, in recent decades the general trend in the number of fires and in the surface burned has increased spectacularly. This increase can be attributed to: (a) land-use changes (Rego 1992; García-Ruiz et al. 1996), and (b) climatic warming (Maheras 1988; Torn and Fried 1992; Amanatidis et al. 1993; Piñol et al. 1998; EPA 2001).

The ecological effects of forest fires are very diverse. This is not only because of the complexity of plant communities and the interface of disturbances such as grazing and cutting with burning, but also because of the different responses to the type, duration and intensity of fire, the season in which it occurs and its frequency (Le Houerou 1987).

The effect of fire on forested ecosystems can range from disastrous to beneficial. Harmful effects include changes in the physical, chemical and biological properties of soils; benefits are the removal of accumulated fuels, an increase in water yield, the control of insects and diseases, the preparation of seedbeds, and the release of seeds from serotinous cones (Kozlowski 2002).

Specifically, the main effects of fire on soils are the loss of nutrients during burning and the increased risk of erosion after burning. The latter is in fact related to the regeneration traits of the previous vegetation and to environmental conditions (Pausas et al. 1999). Large fires that produce a greater number of intensely burned patches can favour the colonization of invasive, fire tolerant species at the expense of rare/endemic species that are less tolerant to post-fire conditions. Thus biodiversity is also affected by fire (Dafis 1990). Climate change predictions and repercussions of forest fires on erosion, water yield and desertification further add to these threats (Moreno et al. 1990).

The effects of forest fires on vegetation are the most evident due to plants vulnerability to burning leading to permanent changes in the composition of the vegetation community, decreased vegetation cover, biomass loss and the alteration of landscape patterns (PerezCabello et al. 2009). However, forest fire is the major force in the biological evolution of biota such as in the Mediterranean (Naveh 1991). For instance, most Mediterranean plant species exhibit effective regeneration mechanisms for overcoming the immediate effects of fire (Mooney and Hobbs 1986). Plant species mechanisms can be passive (e.g. thick insulating bark), or active (e.g. re-sprouting from underground storage organs and seedlings from fire 
protected seeds), which may lead to a rapid process of vegetation cover with similar characteristics to the previous communities (Perez-Cabello et al. 2009). However, the response of vegetation to fire is very complex and it is not easy to generalize because of the large number of factors that can affect the regeneration process (e.g. topographic-climatic influences, plant composition, topographic parameters, soil characteristics, etc.).

Natural regeneration (the regrowth of lost or destroyed parts or organs) of disturbed mature forests to a pre-disturbance condition is often slow, unpredictable, and fraught with difficulties (Kozlowski 2002). The natural regeneration of disturbed forest stands typically occurs in four sequential stages:

1. A stand initiation and regeneration stage: interactions among propagules (including seeds in seed banks and those dispersed into a site as well as sprouting or layering of residual trees) and soil and climatic conditions.

2. A thinning or stem exclusion stage: canopy closes and mortality of trees accelerates, competition for resources (light, water, mineral nutrients), leaf area index reaches its maximum.

3. A transition or understory regeneration stage: death of some overstorey trees, resulting in the formation of gaps in the canopy and the reintroduction of understorey vegetation.

4. A steady-state or old-growth stage: the continuation of a series of successional stages beginning in the previous stage and which may culminate in an old-growth climax forest (Oliver 1981; Oliver and Larson 1996; Kozlowski 2002).

Plant species react to fire through different morphological and physiological traits (PerezCabello et al. 2009). Some can survive fires due to protected plant tissues sprouting (e.g. underground storage organs) and high growth rates after fire and others can rapidly establish seedlings (Buhk et al. 2007). Resprouting ability is a very common survival strategy. Post-fire buds respond by producing new shoots (Miller 2000) and this engenders a rapid return to pre-fire conditions. Differently, seedling establishment may originate from on-site seeds or from off-site seed sources if favourable environmental conditions appear following fire (Baeza and Roy 2008).

The total recovery of a burned area includes different aspects such as revegetation, fauna recuperation, biodiversity, landscape aesthetics, 'natural' runoff rates and sediment yield (Inbar et al. 1998). Opinions about the natural state (the target value of post-fire recovery) differ among disciplines. Seen from a soil and water conservation point of view, a return to the original vegetation cover is sufficient, while ecologists consider recovery as a return in the richness of the original species. From a silvicultural perspective, it is important to consider both the quantity and quality of fire-induced tree regeneration (Gould et al. 2002).

The assessment of the ecological effects of fires on biodiversity, soil degradation and on the cycling of carbon and nitrogen requires not only a detailed and accurate mapping of the burned areas but also an accurate mapping of the type and severity of fire and of post-fire forest regeneration ( for example pine regeneration) and vegetation recovery (for example shrub recovery) (Le Houerou 1987; Jabukauskas et al. 1990; Naveh 1991).

\section{Post-fire monitoring using remote sensing}

This part of the chapter consists of three different sections. Section (3.1) provides information on field based post-fire vegetation monitoring, section (3.2) discusses the airborne and spaceborne sensors used in post-fire monitoring, and section (3.3) introduces 
the relative satellite image analysis techniques. The latter, provides a thoroughly description of well-known methods employed in post-fire monitoring and discuses studies related to the estimation of post-fire albedo and Land Surface Temperature. Subsection (3.3.6) deals with the use of SAR data to monitor the post-fire impact on forests. A summary table (Table 2) is also included.

\subsection{Field based post-fire monitoring}

Traditional methods of recording post-fire impact on vegetation include extensive field work or observations from an airborne platform, followed by the initial mapping (manually) of resource damage into predetermined classes (Bertolette and Spotskey 2001). As fire sizes increase and time becomes a constraining factor, traditional methods have become costly and labour-intensive.

Most studies are carried out in the first years after fire, and are mainly focused on seedling germination and on the survival and the restoration of plant cover (Table 1). Several years after the fire, measurements usually focus more on the characteristics of, for example, the trees, namely height, canopy width, basal diameter and volume. It has been realised that long-term monitoring is often required in order to evaluate the resilience of the different ecosystems towards forest fires. Normally, in long-term post-fire vegetation monitoring sampling units are established right after the fire event and the studied variables are monitored for several consecutive years (Calvo et al. 1998; Tarrega et al. 2001).

The most common sampling technique for monitoring plant populations is the use of permanent square plots. Taking into account both species characteristics and the extent of the study area, the size, the shape and the number of different plots are determined (Arianoutsou 1984; Clemente et al. 1996; Calvo et al. 2002; Cruz et al. 2003; Mitri and Gitas 2010). Accordingly, and in order to facilitate the objective collection of data on the ground, a number of field variables have been measured. In addition, there is a wide variety of sampling protocols depending on the type of survey (Daskalakou and Thanos 2004) that have been developed.

The analysis of post-fire vegetation recovery and monitoring can be either structural (involving variables such as cover and spatial heterogeneity), which is based on visual or floristic assessments (species composition, richness, community diversity, etc.), or a combination of the above (Pausas et al. 1999; Eshel et al. 2000; Kazanis and Arianoutsou 2004). In addition, protocols used can be distinguished into two general categories, that of plots (Thanos et al. 1996; Daskalakou and Thanos 1997; Tsitsoni 1997; Martínez-Sánchez et al. 1999; De Luis et al. 2001; Bailey and Covington 2002; Kennard et al. 2002) and that of transects (Ne'eman et al. 1999; Pausas et al. 1999; Caturla et al. 2000; Wahren et al. 2001; Gould et al. 2002).

Pausas et al. (1999) investigated the effect of different environmental conditions (climatic zones, aspect, and lithology) on the recovery process. They found large spatial variation in plant recovery in different localities with the same aspect, and for different aspects in the same locality. The recovery rate was different for different years due to changing climatic conditions and was higher on north-facing slopes, which are wetter than south-facing slopes. Belda and Meliá (2000) investigated the influence of climate on the natural post-fire regeneration of the burned area and found that regeneration followed an exponential curve, which was stronger in wet zones and had high correlation coefficients. Tsitsoni (1997) found that a high value of organic matter of the burned soil was a favourable factor for natural regeneration, as well as the position on the hillside, with lower regeneration indice values 
for the upper position and increasing values when descending the slope. De Luís et al. (2001) investigated the combined effect of fire and rainstorm on short-term ecosystem response by simulating high intensity rainfall on burned field plots in a Mediterranean shrubland. They found that one-year seedling survival was lower in the plots affected by rainfall simulation than in the control plots. A higher mortality rate, as a consequence of rainfall, was observed in the most abundant species. Pre-fire conditions may also affect the heterogeneity of burn severity, creating a wide range of local and landscape effects (Ne'eman et al. 1999). The propagation of fires and recolonisation processes are events that depend on the spatial organization of vegetation (Mouillet et al. 2001).

\begin{tabular}{|c|c|}
\hline Variables assessed in the field & Reference \\
\hline $\begin{array}{l}\text { Different environmental conditions (climatic } \\
\text { zones, high intensity rainfall, aspect, and } \\
\text { lithology) }\end{array}$ & $\begin{array}{l}\text { (Pausas et al. 1999; Belda and Meliá 2000; De } \\
\text { Luis et al. 2001) }\end{array}$ \\
\hline Organic matter of the burned soil & (Tsitsoni 1997) \\
\hline $\begin{array}{l}\text { Pre-fire conditions (i.e. spatial organisation } \\
\text { of vegetation) }\end{array}$ & $\begin{array}{l}\text { (Calvo et al. 1994; Ne'eman et al. 1999; } \\
\text { Mouillet et al. 2001) }\end{array}$ \\
\hline $\begin{array}{l}\text { Resprouting vigour (plant's anatomical } \\
\text { features, and the characteristics of the } \\
\text { individual before disturbance), intensity of } \\
\text { disturbance, and the environmental } \\
\text { conditions after disturbance }\end{array}$ & $\begin{array}{l}\text { (Lloret and Vilà 1997; Díaz-Delgado and } \\
\text { Pons 2001) }\end{array}$ \\
\hline $\begin{array}{l}\text { Seed banks and germination - Seed number } \\
\text { contained in soil samples - Cone opening } \\
\text { and seed dispersal }\end{array}$ & $\begin{array}{l}\text { (Thanos and Georghiou 1988; Skordilis and } \\
\text { Thanos 1995; Daskalakou and Thanos 1996; } \\
\text { Ferrandis et al. 1996; Herranz et al. 1999; } \\
\text { Keeley 2000) }\end{array}$ \\
\hline $\begin{array}{l}\text { Seedling germination, survival and growth } \\
\text { in a plot and along a transect on which } \\
\text { samples are taken of plant cover, tree cover, } \\
\text { tree characteristics or the floristic } \\
\text { composition and cover per species }\end{array}$ & $\begin{array}{l}\text { (Thanos et al. 1996; Daskalakou and Thanos } \\
\text { 1997; Tsitsoni 1997; Martínez-Sánchez et al. } \\
\text { 1999; Ne'eman et al. 1999; Pausas et al. 1999; } \\
\text { Caturla et al. 2000; De Luis et al. 2001; } \\
\text { Wahren et al. 2001; Bailey and Covington } \\
\text { 2002; Gould et al. 2002; Kennard et al. 2002) }\end{array}$ \\
\hline Post-fire structural dynamics & (Calvo et al. 1991) \\
\hline $\begin{array}{l}\text { Species richness patterns and vegetation } \\
\text { diversity -density of seedlings - abundance } \\
\text { of different age classes }\end{array}$ & $\begin{array}{l}\text { (Pausas et al. 2003; Perula et al. 2003; } \\
\text { Kavgaci et al. 2010) }\end{array}$ \\
\hline $\begin{array}{l}\text { Permanent plot collection per plant } \\
\text { community type and data reduction - Visual } \\
\text { cover of the plant species }\end{array}$ & (Santalla et al. 2002; Clemente et al. 2009) \\
\hline $\begin{array}{l}\text { Non-native species cover correlated with } \\
\text { high native species richness }\end{array}$ & (Hunter et al. 2006) \\
\hline Field spectrometry & $\begin{array}{l}\text { (Broge and Leblanc 2000; Thenkabail et al. } \\
\text { 2000; Thenkabail et al. 2002; Mitri and Gitas } \\
\text { 2010) }\end{array}$ \\
\hline
\end{tabular}

Table 1. Field variables assessed to determine post-fire monitoring 
Vegetative resprouting is possible when buds survive the fire to resprout. Some species regenerate by both sexual and vegetative reproduction. Resprouting vigour depends upon the plant's anatomical features, the characteristics of the individual before disturbance (plant size, number of shoots and physiological status of the plant), intensity of disturbance, and the environmental conditions after disturbance (Lloret and Vilà 1997). Areas where sprouting species are available usually show higher recovery rates than areas with mainly obligated seeders (Díaz-Delgado and Pons 2001). Seed banks are important in the dynamics of many plant communities as they provide an immediate source of propagules for recruitment after disturbance.

Measurements may be focused on the monitoring of seedling germination, survival and growth (height) in a plot (Tsitsoni 1997; Martínez-Sánchez et al. 1999; De Luis et al. 2001; Bailey and Covington 2002; Kennard et al. 2002), or may be along a transect on which samples are taken of plant cover (Caturla et al. 2000), tree cover (Ne'eman et al. 1999), tree characteristics (Ne'eman et al. 1999; Gould et al. 2002) or the floristic composition and cover per species (Pausas et al. 1999; Wahren et al. 2001).

Field spectrometry measurements were employed to investigate the spectral properties of plants, vegetation recovery, and naturally regenerating forest (Broge and Leblanc 2000; Thenkabail et al. 2000; Thenkabail et al. 2002; Mitri and Gitas 2010). More general methods to estimate vegetation abundance (Bonham 1989), recovery and forest regeneration in the field were adopted.

\subsection{Airborne and spaceborne sensors}

In comparison with extensive and labour-intensive field campaigns, remote sensing offers a time- and cost-effective alternative for mapping post-fire vegetation over large areas. Ground truthing based on limited sample sets is, however, always suggested for calibration and validation purposes (Shaw et al. 1998; Mitri and Gitas 2010). Airborne platforms provide a first option to acquire remotely sensed imagery. Stueve et al. (2009) used aerial photography in combination with KH-4B (Key Hole 4B) imagery from the CORONA mission to detect post-fire tree establishment at an alpine treeline ecotone, whereas Amiro et al. (1999) employed airborne measurements to monitor the post-fire energy balance of boreal forest. Peterson and Stow (2003) applied Spectral Mixture Analysis on Airborne Data and Acquisition and Registration (ADAR) data for the mapping of post-fire chaparral regrowth in Southern California. Airborne imagery allows detailed spatial information (Bobbe et al. 2001). However, despite the increasing availability of digital aerial images, these data are rather seldom used. This is explained by the fact that airborne measurements usually cover relatively small areas. As a result, many photographs are required to cover large burned areas, which subsequently require correction and mosaicking (Gitas et al. 2009).

In addition to aerial photographs, spaceborne sensors have shown big potential for assessing post-fire regrowth effects. Table 2 lists a multitude of studies focusing on the use of remote sensing for assessing post-fire vegetation recovery. Satellite sensors are characterized by their technical specifications. These technical specifications determine the sensor's capabilities with regards to the monitoring of post-fire recovery trajectories. In this context, the term resolution is of paramount importance. Resolution is the character of data that limits the user's ability to detect and identify an object of feature within the data (Bobbe et al. 2001). Resolution is fourfold; difference is made between spatial, temporal, spectral and radiometric resolution: 
- Spatial resolution is a measure of fineness of spatial detail and it determines the smallest object that can be identified in the data. For digital sensors, spatial resolution is expressed as pixel size.

- Temporal resolution refers to the sensor's revisiting time, i.e. the time period in which the same area is successively sensed. Temporal resolution depends upon orbital characteristics.

- Spectral resolution is a measure of the specific wavelength intervals in which the sensor records. It is important to distinguish between broadband sensors, in which reflectance values are averaged over relatively wide bandpasses, and hyperspectral sensors, which are characterized by the acquisition over many fine wavebands.

- Radiometric resolution is a measure of the sensor's ability to distinguish between two features of similar reflectance.

Sensor design is a determinant factor when choosing the appropriate image analysis technique. In this context, it is important to trade-off between spatial and temporal resolution. High to moderate spatial resolution (e.g. smaller than $30 \mathrm{~m}$ ) acquire only a few images a year, while low spatial resolution sensors (e.g. larger than $250 \mathrm{~m}$ ) are characterized by daily image acquisition (Veraverbeke et al. 2011a).

Most studies listed in Table 2 are based on optical satellite sensors. These images generally are subject to preprocessing prior to the analysis. In order to obtain geometrically registered top-of-canopy (TOC) reflectance values the imagery usually requires geometric, radiometric, atmospheric and topographic corrections (e.g. Veraverbeke et al. 2010a). After the abrupt changes caused by the fire, the more gradual vegetation recovery leads to alterations in radiometric response at landscape scale. These changes are governed by: (i) disappearance of the charcoal/ash, (ii) changes in the proportion of bare soils and (iii) an increase in vegetative cover. So far, many studies focused on the red-near infrared (R-NIR) bi-spectral space to discriminate between bare soils and vegetated areas because vegetation recovery results in higher NIR reflectance values and lower $\mathrm{R}$ reflectance values due to augmented chlorophyll absorption.

According to the references included in Table 2, applications conducted for post-fire monitoring can be sorted based on the characteristics of the sensor that was used:

- High resolution sensors: 6 studies were based on airborne imagery, 1 on Quickbird imagery and one on $\mathrm{KH}-4 \mathrm{~B}$ imagery.

- Moderate resolutions sensors: the majority of the studies applied Landsat sensors: 27 studies used Thematic Mapper (TM) images, 10 Enhanced Thematic Mapper plus (ETM+) images and 5 Multispectral Scanner images (MSS). In addition, 3 authors applied Satellite Pour l'Observation de la Terra (SPOT) Multispectral (XS) data, 2 Synthetic Aperture Radar (SAR), 1 Earth Observing-1 (EO1) Hyperion and 1 ICESAT Geoscience Laser Altimeter System (GLAS).

- Low resolution sensors: although these sensors are characterized by their low spatial resolution, they have the advantage of repeated temporal sampling with high temporal frequency. Nine studies used Terra Moderate Resolution Imaging Spectroradiometer (MODIS) time series, 5 SPOT Vegetation (VGT) and 5 National Oceanic and Atmospheric Administration (NOAA) Advanced Very High Resolution (AVHRR) Data.

- $\quad$ SAR: three studies used multi-temporal ERS images ( $C$ band), in one study the potential of ALOS PALSAR (L band) was investigated for post-fire monitoring, while multiplepolarization aircraft L-band was used to monitor burn recovery in a coastal marsh. 


\begin{tabular}{|c|c|c|c|c|c|}
\hline Reference & $\begin{array}{c}\text { Ecological } \\
\text { parameter(s) } \\
\text { measured }\end{array}$ & Technique & Ecosystem & Field data & $\begin{array}{c}\text { Remotely } \\
\text { sensed data }\end{array}$ \\
\hline $\begin{array}{c}\text { Alcaraz- } \\
\text { Segura et al. } \\
2010\end{array}$ & Greening & NDVI & Boreal forest & / & AVHRR \\
\hline $\begin{array}{c}\text { Amiro et al. } \\
1999\end{array}$ & $\begin{array}{c}\text { Sensible heat } \\
(\mathrm{H}), \text { latent } \\
\text { heat }(\mathrm{LE}), \\
\mathrm{CO}_{2} \text { flux, } \\
\text { surface } \\
\text { radiometric } \\
\text { temperature } \\
\text { net radiation }\end{array}$ & $\begin{array}{l}\text { Surface } \\
\text { energy } \\
\text { balance }\end{array}$ & Boreal forest & / & $\begin{array}{c}\text { BOREAS } \\
\text { Twin Otter } \\
\text { airborne data }\end{array}$ \\
\hline $\begin{array}{l}\text { Belda and } \\
\text { Melia } 2000\end{array}$ & / & NDVI & $\begin{array}{c}\text { Mediterranean } \\
\text { ecosystem }\end{array}$ & / & $\mathrm{TM}$ \\
\hline $\begin{array}{c}\text { Bisson et al. } \\
2008\end{array}$ & / & NDVI & $\begin{array}{c}\text { Mediterranean } \\
\text { ecosystem }\end{array}$ & Selected plots & TM, ETM+ \\
\hline $\begin{array}{c}\text { Bourgeau- } \\
\text { Chavez et al } \\
2007\end{array}$ & $\begin{array}{c}\text { Soil moisture } \\
\text { variations in } \\
\text { fire disturbed } \\
\text { areas }\end{array}$ & $\begin{array}{l}\text { Regression } \\
\text { models }\end{array}$ & Boreal forest & $\begin{array}{l}510 X 10 \\
\text { sample plots, } \\
\text { randomly } \\
\text { selected along } \\
200 \mathrm{~m} \text { long } \\
\text { transcects } \\
\end{array}$ & ERS 2 \\
\hline $\begin{array}{c}\text { Carranza et } \\
\text { al. } 2001\end{array}$ & / & $\begin{array}{l}\text { NDVI and } \\
\text { landscape } \\
\text { structure }\end{array}$ & $\begin{array}{c}\text { Mediterranean } \\
\text { ecosystem }\end{array}$ & $\begin{array}{c}33 \text { line } \\
\text { transect plots }\end{array}$ & TM \\
\hline $\begin{array}{l}\text { Clemente et } \\
\text { al. } 2009\end{array}$ & $\begin{array}{l}\text { Fractional } \\
\text { vegetation } \\
\text { cover }\end{array}$ & $\begin{array}{l}\text { NDVI and } \\
\text { other indices }\end{array}$ & $\begin{array}{c}\text { Mediterranean } \\
\text { ecosystem }\end{array}$ & / & TM, ETM+ \\
\hline $\begin{array}{c}\text { Cuevas- } \\
\text { Gonzalez et } \\
\text { al. } 2008\end{array}$ & fAPAR & $\begin{array}{c}\text { NDVI } \\
\text { transformation }\end{array}$ & Boreal forest & / & MODIS \\
\hline $\begin{array}{c}\text { Cuevas- } \\
\text { Gonzalez et } \\
\text { al. } 2009\end{array}$ & / & $\begin{array}{l}\text { NDVI and } \\
\text { other indices }\end{array}$ & Boreal forest & / & MODIS \\
\hline $\begin{array}{c}\text { Diaz- } \\
\text { Delgado and } \\
\text { Pons } 2001 \\
\end{array}$ & / & $\begin{array}{l}\text { NDVI and } \\
\text { control plot } \\
\text { selection }\end{array}$ & $\begin{array}{c}\text { Mediterranean } \\
\text { ecosystem }\end{array}$ & / & MSS \\
\hline $\begin{array}{c}\text { Diaz- } \\
\text { Delgado et } \\
\text { al. } 2002\end{array}$ & / & $\begin{array}{l}\text { NDVI and } \\
\text { control plot } \\
\text { selection }\end{array}$ & $\begin{array}{c}\text { Mediterranean } \\
\text { ecosystem }\end{array}$ & / & MSS \\
\hline $\begin{array}{c}\text { Diaz- } \\
\text { Delgado et } \\
\text { al. } 2003 \\
\end{array}$ & / & $\begin{array}{l}\text { NDVI and } \\
\text { control plot } \\
\text { selection }\end{array}$ & $\begin{array}{c}\text { Mediterranean } \\
\text { ecosystem }\end{array}$ & / & MSS, TM \\
\hline
\end{tabular}




\begin{tabular}{|c|c|c|c|c|c|}
\hline Reference & $\begin{array}{c}\text { Ecological } \\
\text { parameter(s) } \\
\text { measured }\end{array}$ & Technique & Ecosystem & Field data & $\begin{array}{c}\text { Remotely } \\
\text { sensed data }\end{array}$ \\
\hline $\begin{array}{c}\text { Goetz et al. } \\
2006\end{array}$ & / & NDVI & Boreal forest & 11 sites & AVHRR \\
\hline $\begin{array}{c}\text { Goetz et al. } \\
2010\end{array}$ & Tree height & LIDAR & Boreal forest & / & $\begin{array}{c}\text { GLAS, TM, } \\
\text { MODIS }\end{array}$ \\
\hline $\begin{array}{c}\text { Gouveia et } \\
\text { al. } 2010\end{array}$ & / & NDVI & $\begin{array}{c}\text { Mediterranean } \\
\text { ecosystem }\end{array}$ & / & VGT \\
\hline $\begin{array}{c}\text { Hall et al. } \\
1991\end{array}$ & $\begin{array}{l}\text { Land cover } \\
\text { classification } \\
\text { including } \\
\text { regeneration } \\
\text { classes }\end{array}$ & $\begin{array}{c}\text { Supervised } \\
\text { classification }\end{array}$ & Boreal forest & / & MSS \\
\hline $\begin{array}{l}\text { Henry and } \\
\text { Hope } 1998\end{array}$ & / & $\begin{array}{l}\text { NDVI and } \\
\text { other indices }\end{array}$ & $\begin{array}{c}\text { Mediterranean } \\
\text { ecosystem }\end{array}$ & $\begin{array}{c}36 \text { line } \\
\text { transect plots } \\
\text { with plant } \\
\text { community } \\
\text { statistics }\end{array}$ & XS \\
\hline $\begin{array}{l}\text { Hernandez- } \\
\text { Clemente et } \\
\text { al. } 2009\end{array}$ & / & NDVI & $\begin{array}{c}\text { Mediterranean } \\
\text { ecosystem }\end{array}$ & / & TM, ETM+ \\
\hline $\begin{array}{c}\text { Hicke et al. } \\
2003\end{array}$ & NPP & $\begin{array}{c}\text { NDVI } \\
\text { transformation }\end{array}$ & Boreal forest & / & AVHRR \\
\hline $\begin{array}{c}\text { Hope et al. } \\
2007\end{array}$ & / & NDVI & $\begin{array}{c}\text { Mediterranean } \\
\text { ecosystem }\end{array}$ & / & TM, ETM+ \\
\hline $\begin{array}{l}\text { Idris et al. } \\
2005\end{array}$ & / & $\begin{array}{l}\text { NDVI and } \\
\text { control plot } \\
\text { selection }\end{array}$ & $\begin{array}{l}\text { Tropical and } \\
\text { boreal forest }\end{array}$ & / & AVHRR \\
\hline $\begin{array}{c}\text { Jabukauskas } \\
\text { et al. } 1990\end{array}$ & $\begin{array}{l}\text { Land cover } \\
\text { classification } \\
\text { including } \\
\text { regeneration } \\
\text { classes }\end{array}$ & $\begin{array}{l}\text { Supervised } \\
\text { minimum } \\
\text { distance-to- } \\
\text { mean } \\
\text { classification }\end{array}$ & $\begin{array}{c}\text { Temperate } \\
\text { coniferous } \\
\text { forest }\end{array}$ & $\begin{array}{c}32 \text { linguistic } \\
\text { sample points }\end{array}$ & $\mathrm{TM}$ \\
\hline $\begin{array}{c}\text { Jacobson } \\
2010\end{array}$ & / & $\begin{array}{l}\text { NDVI and } \\
\text { other indices }\end{array}$ & $\begin{array}{l}\text { Woodland } \\
\text { community }\end{array}$ & / & XS \\
\hline $\begin{array}{c}\text { Kasischke et } \\
\text { al } 2007\end{array}$ & $\begin{array}{l}\text { Relations } \\
\text { between soil } \\
\text { moisture } \\
\text { patterns and } \\
\text { post-fire tree } \\
\text { recruitment }\end{array}$ & $\begin{array}{l}\text { Empirical } \\
\text { relations }\end{array}$ & Boreal forest & 2 test sites & ERS 1 and 2 \\
\hline
\end{tabular}




\begin{tabular}{|c|c|c|c|c|c|}
\hline Reference & $\begin{array}{c}\text { Ecological } \\
\text { parameter(s) } \\
\text { measured }\end{array}$ & Technique & Ecosystem & Field data & $\begin{array}{c}\text { Remotely } \\
\text { sensed data }\end{array}$ \\
\hline $\begin{array}{c}\text { Kasischke et } \\
\text { al } 2011\end{array}$ & $\begin{array}{c}\text { Biomass } \\
\text { estimation of } \\
\text { regenerating } \\
\text { forests }\end{array}$ & $\begin{array}{l}\text { Empirical } \\
\text { relations }\end{array}$ & Boreal forest & / & $\begin{array}{c}\text { ALOS } \\
\text { PALSAR }\end{array}$ \\
\hline $\begin{array}{l}\text { Lhermitte et } \\
\text { al. } 2010\end{array}$ & / & $\begin{array}{l}\text { NDVI and } \\
\text { control plot } \\
\text { selection }\end{array}$ & $\begin{array}{l}\text { Savannah } \\
\text { ecosystem }\end{array}$ & / & VGT \\
\hline $\begin{array}{l}\text { Lhermitte et } \\
\text { al. } 2011\end{array}$ & / & $\begin{array}{l}\text { NDVI and } \\
\text { control plot } \\
\text { selection }\end{array}$ & $\begin{array}{l}\text { Savannah } \\
\text { ecosystem }\end{array}$ & / & VGT \\
\hline Li et al. 2008 & / & $\begin{array}{c}\text { NDVI, EVI } \\
\text { and control } \\
\text { plot selection }\end{array}$ & $\begin{array}{l}\text { Temperate } \\
\text { forest }\end{array}$ & / & MODIS \\
\hline $\begin{array}{l}\text { Lozano et al. } \\
\qquad 2010\end{array}$ & / & $\begin{array}{l}\text { NDVI and } \\
\text { other indices, } \\
\text { landscape } \\
\text { structure }\end{array}$ & $\begin{array}{c}\text { Mediterranean } \\
\text { ecosystem }\end{array}$ & / & TM, ETM+ \\
\hline $\begin{array}{c}\text { Lyons et al. } \\
2008\end{array}$ & / & Albedo & Boreal forest & / & MODIS \\
\hline $\begin{array}{c}\text { Malak and } \\
\text { Pausas } 2006\end{array}$ & / & NDVI & \begin{tabular}{|c}
$\begin{array}{c}\text { Mediterranean } \\
\text { ecosystem }\end{array}$ \\
\end{tabular} & / & $\mathrm{TM}$ \\
\hline $\begin{array}{c}\text { Marchetti et } \\
\text { al. } 1995\end{array}$ & / & Infrared index & \begin{tabular}{|c|}
$\begin{array}{c}\text { Mediterranean } \\
\text { ecosystem }\end{array}$ \\
\end{tabular} & / & TM \\
\hline $\begin{array}{c}\text { McMichael } \\
\text { et al. } 2004\end{array}$ & LAI & $\begin{array}{c}\text { NDVI } \\
\text { transformation }\end{array}$ & $\begin{array}{c}\text { Mediterranean } \\
\text { ecosystem }\end{array}$ & $\begin{array}{l}62 \text { sample } \\
\text { points and } \\
\text { field } \\
\text { spectroscopy }\end{array}$ & TM, ETM+ \\
\hline $\begin{array}{l}\text { Minchella et } \\
\text { al. } 2009\end{array}$ & $\begin{array}{l}\text { Vegetation } \\
\text { recovery }\end{array}$ & $\begin{array}{l}\text { Simulations } \\
\text { with } \\
\text { scattering } \\
\text { model }\end{array}$ & $\begin{array}{c}\text { Mediterranean } \\
\text { ecosystem }\end{array}$ & / & ERS \\
\hline $\begin{array}{c}\text { Mitchell and } \\
\text { Yuan } 2010 \\
\end{array}$ & / & NDVI & $\begin{array}{c}\begin{array}{c}\text { Temperate } \\
\text { forest }\end{array} \\
\end{array}$ & / & $\mathrm{TM}$ \\
\hline $\begin{array}{l}\text { Mitri and } \\
\text { Gitas } 2010\end{array}$ & $\begin{array}{l}\text { Land cover } \\
\text { classification } \\
\text { including } \\
\text { regeneration } \\
\text { classes } \\
\end{array}$ & $\begin{array}{l}\text { Object-based } \\
\text { classification }\end{array}$ & $\begin{array}{c}\text { Mediterranean } \\
\text { ecosystem }\end{array}$ & / & Hyperion \\
\hline $\begin{array}{l}\text { Palandjian et } \\
\text { al. } 2009\end{array}$ & $\begin{array}{l}\text { Classification } \\
\text { of } 4 \\
\text { regeneration } \\
\text { classes }\end{array}$ & $\begin{array}{c}\text { Density } \\
\text { slicing on } \\
\text { NDVI data }\end{array}$ & $\begin{array}{c}\text { Mediterranean } \\
\text { ecosystem }\end{array}$ & Flux tower & Quickbird \\
\hline
\end{tabular}




\begin{tabular}{|c|c|c|c|c|c|}
\hline Reference & \begin{tabular}{|c|} 
Ecological \\
parameter(s) \\
measured
\end{tabular} & Technique & Ecosystem & Field data & $\begin{array}{c}\text { Remotely } \\
\text { sensed data }\end{array}$ \\
\hline $\begin{array}{c}\text { Peterson and } \\
\text { Stow } 2003\end{array}$ & $\begin{array}{l}\text { Fractional } \\
\text { vegetation } \\
\text { cover }\end{array}$ & SMA & $\begin{array}{c}\text { Mediterranean } \\
\text { ecosystem }\end{array}$ & / & TM, ADAR \\
\hline $\begin{array}{c}\text { Ramsey et al. } \\
1999\end{array}$ & $\begin{array}{c}\text { Time-since- } \\
\text { burn }\end{array}$ & $\begin{array}{l}\text { Regression } \\
\text { analysis }\end{array}$ & $\begin{array}{c}\text { Marsh } \\
\text { ecosystem }\end{array}$ & / & Aircraft SAR \\
\hline $\begin{array}{l}\text { Randerson et } \\
\text { al. } 2006\end{array}$ & / & Albedo & Boreal forest & $\begin{array}{c}93 \text { line } \\
\text { transect } \\
\text { points and } \\
\text { field } \\
\text { spectroscopy }\end{array}$ & MODIS \\
\hline $\begin{array}{l}\text { Riaño et al. } \\
2002\end{array}$ & / & $\begin{array}{l}\text { NDVI and } \\
\text { control plot } \\
\text { selection }\end{array}$ & $\begin{array}{c}\text { Mediterranean } \\
\text { ecosystem }\end{array}$ & / & AVIRIS \\
\hline $\begin{array}{c}\text { Ricotta et al. } \\
1998\end{array}$ & / & $\begin{array}{l}\text { NDVI and } \\
\text { landscape } \\
\text { structure }\end{array}$ & $\begin{array}{c}\text { Mediterranean } \\
\text { ecosystem }\end{array}$ & / & $\mathrm{TM}$ \\
\hline $\begin{array}{c}\text { Roder et al. } \\
2008\end{array}$ & $\begin{array}{l}\text { Fractional } \\
\text { vegetation } \\
\text { cover }\end{array}$ & SMA & $\begin{array}{c}\text { Mediterranean } \\
\text { ecosystem }\end{array}$ & $\begin{array}{c}\text { Ground- } \\
\text { based NDVI }\end{array}$ & $\begin{array}{c}\text { MSS, TM, } \\
\text { ETM+ }\end{array}$ \\
\hline $\begin{array}{c}\text { Sankey et al. } \\
2008\end{array}$ & $\begin{array}{l}\text { Fractional } \\
\text { vegetation } \\
\text { cover }\end{array}$ & SMA & $\begin{array}{l}\text { Sagebrush } \\
\text { community }\end{array}$ & $\begin{array}{c}\text { Field } \\
\text { spectroscopy }\end{array}$ & $\begin{array}{c}\text { XS, aerial } \\
\text { photographs }\end{array}$ \\
\hline $\begin{array}{c}\text { Schroeder } \\
\text { and Pereira } \\
2002\end{array}$ & / & $\begin{array}{l}\text { Landscape } \\
\text { structure }\end{array}$ & Boreal forest & / & $\mathrm{TM}$ \\
\hline $\begin{array}{c}\text { Segah et al. } \\
2010\end{array}$ & / & NDVI & Tropical forest & $\begin{array}{c}60 \text { sample } \\
\text { points }\end{array}$ & TM, VGT \\
\hline $\begin{array}{c}\text { Shaw et al. } \\
1998\end{array}$ & / & $\begin{array}{l}\text { NDVI and } \\
\text { other indices }\end{array}$ & Boreal forest & / & $\begin{array}{c}\text { Field } \\
\text { spectroscopy }\end{array}$ \\
\hline $\begin{array}{l}\text { Steyaert et } \\
\text { al. } 1997\end{array}$ & $\begin{array}{l}\text { Land cover } \\
\text { classification } \\
\text { including } \\
\text { regeneration } \\
\text { classes }\end{array}$ & $\begin{array}{l}\text { Unsupervised } \\
\text { cluster } \\
\text { classification }\end{array}$ & Boreal forest & / & AVHRR, TM \\
\hline $\begin{array}{c}\text { Stueve et al. } \\
2009\end{array}$ & $\begin{array}{c}\text { Tree } \\
\text { establishment } \\
\text { classification }\end{array}$ & $\begin{array}{l}\text { Supervised } \\
\text { minimum } \\
\text { distance-to- } \\
\text { mean binary } \\
\text { classification }\end{array}$ & $\begin{array}{l}\text { Alpine } \\
\text { treeline } \\
\text { border }\end{array}$ & $\begin{array}{l}\text { Line transect } \\
\text { plots }\end{array}$ & $\begin{array}{c}\text { Airborne } \\
\text { and KH-4B } \\
\text { imagery }\end{array}$ \\
\hline
\end{tabular}




\begin{tabular}{|c|c|c|c|c|c|}
\hline Reference & $\begin{array}{c}\text { Ecological } \\
\text { parameter(s) } \\
\text { measured }\end{array}$ & Technique & Ecosystem & Field data & $\begin{array}{c}\text { Remotely } \\
\text { sensed data }\end{array}$ \\
\hline $\begin{array}{c}\text { Telesca and } \\
\text { Lasaponara } \\
2006\end{array}$ & / & $\begin{array}{c}\text { Detrended } \\
\text { fluctuation } \\
\text { analysis on } \\
\text { NDVI time } \\
\text { series }\end{array}$ & $\begin{array}{c}\text { Mediterranean } \\
\text { ecosystem }\end{array}$ & $\begin{array}{l}78 \text { line } \\
\text { transect plots } \\
\text { and field } \\
\text { spectroscopy }\end{array}$ & VGT \\
\hline $\begin{array}{c}\text { Van } \\
\text { Leeuwen } \\
2008 \\
\end{array}$ & $\begin{array}{l}\text { Phenological } \\
\text { metrics }\end{array}$ & NDVI & $\begin{array}{l}\text { Temperate } \\
\text { ecosystem }\end{array}$ & / & MODIS \\
\hline $\begin{array}{l}\text { Van } \\
\text { Leeuwen et } \\
\text { al. } 2010\end{array}$ & $\begin{array}{l}\text { Phenological } \\
\text { metrics }\end{array}$ & $\begin{array}{l}\text { NDVI and } \\
\text { control plot } \\
\text { selection }\end{array}$ & $\begin{array}{c}\text { Temperate } \\
\text { and } \\
\text { Mediterranean } \\
\text { ecosystems }\end{array}$ & $\begin{array}{c}78 \text { line } \\
\text { transect plots } \\
\text { and field } \\
\text { spectroscopy }\end{array}$ & MODIS \\
\hline $\begin{array}{c}\text { Veraverbeke } \\
\text { et al. } 2012\end{array}$ & $\begin{array}{l}\text { Fractional } \\
\text { vegetation } \\
\text { cover }\end{array}$ & $\begin{array}{l}\text { NDVI and } \\
\text { other indices }\end{array}$ & $\begin{array}{c}\text { Mediterranean } \\
\text { ecosystem }\end{array}$ & / & TM \\
\hline $\begin{array}{c}\text { Veraverbeke } \\
\text { et al. } 2012\end{array}$ & / & $\begin{array}{l}\text { NDVI, albedo, } \\
\text { LST and } \\
\text { control plot } \\
\text { selection }\end{array}$ & $\begin{array}{c}\text { Mediterranean } \\
\text { ecosystem }\end{array}$ & / & MODIS \\
\hline $\begin{array}{c}\text { Veraverbeke } \\
\text { et al. } 2012\end{array}$ & $\begin{array}{l}\text { Fractional } \\
\text { vegetation } \\
\text { cover }\end{array}$ & SMA & $\begin{array}{c}\text { Mediterranean } \\
\text { ecosystem }\end{array}$ & $\begin{array}{l}19 \text { line } \\
\text { transect } \\
\text { points }\end{array}$ & TM \\
\hline $\begin{array}{c}\text { Vicente- } \\
\text { Serrano et al. } \\
2008\end{array}$ & / & $\begin{array}{l}\text { NDVI and } \\
\text { other indices }\end{array}$ & $\begin{array}{c}\text { Mediterranean } \\
\text { ecosystem }\end{array}$ & / & TM/ETM+ \\
\hline $\begin{array}{c}\text { Viedma et al. } \\
1997\end{array}$ & $\begin{array}{l}\text { Regeneration } \\
\text { rate }\end{array}$ & NDVI & $\begin{array}{c}\text { Mediterranean } \\
\text { ecosystem }\end{array}$ & / & TM \\
\hline $\begin{array}{c}\text { Vila and } \\
\text { Barbosa } 2010\end{array}$ & $\begin{array}{l}\text { Fractional } \\
\text { vegetation } \\
\text { cover }\end{array}$ & $\begin{array}{c}\text { NDVI and } \\
\text { other indices, } \\
\text { SMA }\end{array}$ & $\begin{array}{c}\text { Mediterranean } \\
\text { ecosystem }\end{array}$ & Field data & TM, ETM+ \\
\hline $\begin{array}{c}\text { White et al. } \\
1996\end{array}$ & / & NDVI & Boreal forest & / & $\mathrm{TM}$ \\
\hline $\begin{array}{c}\text { Wittenberg } \\
\text { et al. } 2007\end{array}$ & / & EVI & $\begin{array}{c}\text { Mediterranean } \\
\text { ecosystem }\end{array}$ & / & TM, ETM+ \\
\hline
\end{tabular}

(ADAR: Airborne Data and Acquisition and Registration, AVHRR: Advanced Very High Resolution Radiometer, AVIRIS: Airborne Visible/Infrared Imaging Spectroradiometer, ETM+: Enhanced Thematic Mapper plus, EVI: Enhanced Vegetation Index, fAPAR: fraction of Absorbed Photosynthetically Active Radiation, GLAS: Geoscience Laser Altimeter System, KH: Key Hole, LAI: Leaf Area Index, LST: Land Surface Temperature, LIDAR, Light Detection and Ranging)MSS: Multispectral Scanner, MODIS: Moderate Resolution Imaging Spectroradiometer, NDVI: Normalized Difference Vegetation Index, NPP: Net Primary Productivity, RADAR: Radio Detection and Ranging, SAR: Synthetic Aperture Radar, SMA: Spectral Mixture Analysis, TM: Thematic Mapper, XS: Multispectral VGT: Vegetation

Table 2. Examples of post-fire vegetation recovery studies using remotely sensed data 
A review of the studies presented in Table 2, shows that current research mainly focused on multispectral Landsat, MODIS, VGT and AVHRR data. The moderate and low resolution scales are relatively well documented. For the coarse spatial resolution this usually goes hand in hand with time series analysis. The overview also highlights a need for further exploration of high spatial resolution and high spectral resolution, i.e. hyperspectral, data. High resolution data (e.g. Quickbird, IKONOS) could help in the recognition of individual plants, whereas hyperspectral data have the potential to discriminate between different vegetation species (Asner and Lobell 2000; Mitri and Gitas 2010; Somers et al. 2010). Besides optical imagery, Synthetic Aperture Radar (SAR) data is also worth a more in-depth evaluation.

From Table 2 it can also be concluded that vegetation recovery studies have been carried out in a number of different ecosystems including:

- Mediterranean ecosystems: 26 studies were conducted in the Mediterranean basin, whereas 5 papers concentrate on the Mediterranean ecozone of California (USA).

- Boreal forests: 20 study areas were carried out in boreal forests of North American and the Eurasian boreal zone.

- Temperate forests: 5 studies focused on temperate forests..

- Other types of forests: 2 studies were conducted in tropical forests, 2 in savannah ecosystems, 1 along an alpine treeline border, 1 in a sagebrush community and 1 in a marsh ecosystem.

Post-fire recovery rates depend on fire severity (Diaz-Delgado et al. 2003), soil properties (Bisson et al. 2008), post-fire meteorological conditions (Henry and Hope 1998; van Leeuwen et al. 2010) and ecotype (Viedma et al. 1997; Veraverbeke et al. 2010b; Lhermitte et al. 2011; Veraverbeke et al. 2011b). In fire-adapted sclerophyllous shrub lands, for example, recovery only takes a few years (Viedma et al. 1997; Pausas and Verdu 2005) whereas in boreal forests recovery lasts several decades (Nepstad et al. 1999). The summary above clearly shows that recovery research so far focused on boreal and Mediterranean ecosystems. Table 2 also reveals that only $26 \%$ of the papers included in the list were supported by ground truth. This highlights a need to conduct research on the remote sensing of post-fire vegetation recovery supported by field campaigns, while other ecosystems such as tropical forest and savannah ecosystems urgently require a knowledge gain.

\subsection{Image analysis techniques}

Several image analysis techniques are employed in the remote sensing of post-fire recovery. Most of the traditional approaches have an origin outside fire applications but their methodology is easily adjustable for recovery studies. The most important traditional methods are image classification, Vegetation Indices (VIs) and Spectral Mixture Analysis (SMA). They are thoroughly discussed below. In addition, one specific technique applicable for ecological disturbances, i.e. control plot selection, is also incorporated. Finally, some less frequently used approaches are shortly described.

\subsubsection{Image classification}

Since long, multispectral image classification is known to be a powerful technique to translate remotely sensed data into ecologically relevant cover classes. Both supervised (Jakubauskas et al. 1990; Hall et al. 1991; Stueve et al. 2009; Mitri and Gitas 2010) and unsupervised (Steyaert et al. 1997) techniques have been applied in post-fire recovery studies. Most applications rely on pixel-based classifiers such as the minimum-distance- 
to-mean (Jakubauskas et al. 1990; Stueve et al. 2009) and maximum likelihood classifiers (Hall et al. 1991). While the majority of the studies focused on four or more cover classes (Jabukauskas et al. 1990; Hall et al. 1991; Steyaert et al. 1997; Mitri and Gitas 2010), the study of Stueve et al. (2009) was restricted to a binary classification of tree establishment along an alpine treeline border. A major problem associated with pixel-based classifications is the occurrence of salt-and-pepper artifacts. As a solution, object-based classification schemes include both spectral and contextual information (Wicks et al. 2002) (See more in Section 4).

Apart from multispectral classification approaches Palandjian et al. (2009) applied density slicing on post-fire Normalized Difference Vegetation Index (NDVI) data. As such, they discriminated four different regeneration classes. Generally spoken, relatively few studies applied image classification to monitor post-fire recovery. This is mainly explained by the fact that the spatial resolution of most popular satellite systems (e.g. Landsat) exceeds the size of individual regenerating plants. As a result, it is very difficult in these applications to find pure training data because most image pixels are mixed. This incites a need to explore the potential of high resolution imagery (e.g. Quickbird, IKONOS) to classify individual plants. This would also open new research pathways to study the small-scale spatial patterns of post-fire vegetation recovery.

\subsubsection{Vls}

By far the most widely used remote sensing technique to assess post-fire recovery is the NDVI (Tucker 1979) because of its strongly established relationship with above-ground biomass in a wide range of ecosystems (Carlson and Ripley 1997; Henry and Hope 1998; Cuevas-Gonzalez et al. 2009). The post-fire environment typically consists of a mixture of vegetation and substrate. Theoretically, Soil Adjusted Vegetation Indices (SAVIs) (Huete 1988; Baret and Guyot 1991; Qi et al. 1994) are better suited for these mixed environments. Relatively few studies have assessed the correlation between field estimates of vegetative cover and VIs. Clemente et al. (2009) contrasted the NDVI with the SAVI (Huete 1988), Transformed SAVI (TSAVI), (Baret and Guyot 1991) and Modified SAVI (MSAVI) (Qi et al. 1994) for estimating post-fire vegetation regrowth 7 and 12 years after a fire in Spain. The NDVI was stronger related to field estimates of vegetation cover than any other index. Vila and Barbosa (2010) drew more or less the same conclusion. They also found that the NDVI was most accurately related to field data eight years after a fire in Italy. Van Leeuwen et al. (2010) also retrieved high correlations between NDVI and field data of recovery. Veravrbeke et al. (2012b) comprehensively evaluated thirteen R-NIR vegetation indices for assessing post-fire vegetation recovery. They found that the NDVI indeed obtained the best correlations with line transect field data and the failure of the SAVIs was due to their inability to account for variations in background brightness. This approves the use of the NDVI as an appropriate recovery measure, however, it should be noted that the potential of spectral indices with a SWIR or MIR spectral band has not been fully explored yet. These spectral regions have proven to be very effective in discriminating soil and vegetation (Drake et al. 1999; Asner and Lobell 2000). In the context of post-fire recovery, VIs including a SWIR or MIR band have shown prospect in the studies of Marchetti et al. (1995), CuevasGonzalez et al. (2009) and Jacobson (2010).

Several studies used NDVI data as a linkage to more ecologically relevant parameters such as fractional vegetation cover (Clemente et al. 2009; Vila and Barbosa 2010; Veraverbeke et 
al. 2012b), fraction of Absorbed Photosynthetically Active Radiation (fAPAR), (CuevasGonzalez et al. 2008), Net Primary Production (NPP), (Hicke et al. 2003) and Leaf Area Index (LAI), (McMichael et al. 2004). Usually, these index transforms are based on thorough field calibration. Only few studies incorporated a substantial number of field plots to calibrate and validate the VI approach (Shaw et al. 1998; Bisson et al. 2008; Clemente et al. 2009; Jacobson 2010; Segah et al. 2010; van Leeuwen et al. 2010; Vila and Barbosa 2010; Veraverbeke et al. 2011b Hernandez-Clemente et al. 2009). Figure 1 presents an example of the relationship between the NDVI and field estimates of vegetative cover which was used to model post-fire vegetation cover in the burned area. The majority of the authors, however, use the NDVI as a well-accepted methodology without additional field efforts. In a mono-temporal context NDVI were related to climatic variables (Belda and Meliá 2000), topographic parameters (Mitchell and Yuan 2010) and fire severity (White et al. 1996; Mitchell and Yuan 2010).

Other studies employed multiple images to construct recovery trajectories (Viedma et al. 1997; Henry and Hope 1998; Ricotta et al. 1998; Carranza et al. 2001; Díaz-Delgado and Pons 2001; Diaz-Delgado et al. 2002; Riaño et al. 2002; Malak and Pausas 2006; Wittenberg et al. 2007; Bisson et al. 2008; Vicente-Serrano et al. 2008; Clemente et al. 2009; HernandezClemente et al. 2009). Where Landsat-based studies allow only a few cloud-free images a year (Ju and Roy 2008), satellite sensors with high temporal frequency permit the construction of continuous time series. More recently, several authors have explored this data type for assessing post-fire effects (Idris et al. 2005; Goetz et al. 2006; Telesca and Lasaponara 2006; Li et al. 2008; van Leeuwen 2008; Alcaraz-Segura et al. 2010; Gouveia et al. 2010; Lhermitte et al. 2010; Segah et al. 2010; van Leeuwen et al. 2010; Lhermitte et al. 2011; Veraverbeke et al. 2012a; Veraverbeke et al. 2012c). Thanks to this it is possible to discriminate between regeneration patters and seasonal fluctuations (Veraverbeke et al. 2010b; Lhermitte et al. 2011; Veraverbeke et al. 2012b). A major advantage of multi-temporal data is that regression fits between time since fire and NDVI data give reliable recovery rate estimates (Viedma et al. 1997; Díaz-Delgado and Pons 2001; Gouveia et al. 2010). Extension of these regeneration rates allows prediction on the future state of biomass. This is of major interest for decision makers in rangeland management. NDVI data also served as the preferred data source for the control plot selection procedure (Díaz-Delgado and Pons 2001; Diaz-Delgado et al. 2002; Lhermitte et al. 2010; Lhermitte et al. 2011) and landscape ecological applications (Ricotta et al. 1998; Carranza et al. 2001).

\subsubsection{Spectral mixture analysis}

The post-fire environment typically consists of a mixture of vegetation and substrate. Thus, monitoring post-fire regeneration processes essentially poses a sub-pixel issue at the resolution of most operational satellite systems such as Landsat. A number of image analysis techniques accommodating mixing problems exist (Atkinson et al. 1997; Arai 2008) with SMA being the most common technique utilized in many applications (Roberts et al. 1998; Asner and Lobell 2000; Riaño et al. 2002; Roder et al. 2008; Somers et al. 2010). SMA effectively addresses this issue by quantifying the sub-pixel fraction of cover of different endmembers, which are assumed to represent the spectral variability among the dominant terrain features. A major advantage of SMA is its ability to detect low cover fractions, something which remains difficult with the traditional vegetation indices (VIs) approach (Henry and Hope 1998; Elmore et al. 2000; Rogan and Franklin 2001). Moreover, SMA 
A.



B.



Fig. 1. Fractional vegetation cover map (A) three years after the large Peloponnese (Greece) fires based on the regression fit between the Landsat Normalized Difference Vegetation Index (NDVI) and line transect field ratings of vegetation cover (B) (Veraverbeke et al. 2012b). 
directly results in quantitative abundance maps, without the need of an initial calibration based on field data as with VIs (Somers et al. 2010; Vila and Barbosa 2010). With regards to post-fire effects, rather few studies employed SMA to monitor post-fire vegetation responses (Riaño et al. 2002; Peterson and Stow 2003; Roder et al. 2008; Sankey et al. 2008; Vila and Barbosa 2010; Veraverbeke et al. 2012a). Although results of these studies were consistent, most of them were all restricted to simple linear SMA models in which only one spectrum was allowed for each endmember. As a consequence, the performance of these SMA models often appeared to be suboptimal (Roder et al. 2008; Vila and Barbosa 2010) because these models did not incorporate the natural variability in scene conditions of terrain features inherent in remote sensing data (Asner 1998). To overcome this variability effect Peterson and Stow (2003) applied multiple endmember SMA (MESMA), (Roberts et al. 1998). MESMA incorporates natural variability by allowing multiple endmembers for each constituting terrain feature. These endmember sets represent the within-class variability (Somers et al. 2010) and MESMA models search for the most optimal endmember combination by reducing the residual error when estimating fractional covers (Asner and Lobell 2000). Rogge et al. (2006) and Veraverbeke et al. (2012a), however, clearly demonstrated that reducing the residual error by applying MESMA not always results in the selection of the most appropriate endmember spectrum. An initial segmentation of the area prior to the unmixing process in order to retain areas which reveal a high similarity in the spectral properties of a certain endmember has been presented as a sound and computationally efficient solution to address this issue (Rogge et al. 2006; Veraverbeke et al. 2012a).

A possible amelioration in post-fire vegetation mapping using SMA could be the inclusion of SWIR and MIR spectral regions in the unmixing process. These spectral regions have proven to be very effective in discriminating soil and vegetation (Drake et al. 1999; Asner and Lobell 2000). Carreiras et al. (2006) demonstrated that adding the SWIR-MIR Landsat bands resulted in better estimates of tree canopy cover in Mediterranean shrublands. Additionally, enhancing the spectral resolution by employing hyperspectral data would increase the amount of spectral detail which would benefit the differentiation between spectra (Mitri and Gitas 2008). By including more and other spectral wavebands the unmixing model could gain discriminative power. Potentially, this would make it even possible to distinguish between non-photosynthetic vegetation and substrate (Asner and Lobell 2000; Somers et al. 2010), which appeared to be impossible in current applications.

\subsubsection{Control plot selection}

A major difficulty in post-fire time series analysis is that the analysis can be hampered by phenological effects, both due to the differences in acquisition data and due to inter-annual meteorological variability (Díaz-Delgado and Pons 2001). To deal with these phenological effects Diaz-Delgado and Pons (2001) proposed to compare vegetation regrowth in a burned area with unburned reference plots within the same image. As such, external and phenological variations are minimized among the compared areas. Several authors have successfully adapted the reference plot approach (Diaz-Delgado et al. 2002; Diaz-Delgado et al. 2003; Idris et al. 2005; Li et al. 2008; van Leeuwen et al. 2010). The reference plot selection procedure has, however, two main difficulties. Firstly, large scale application remains constrained due to the necessity of profound field knowledge to select relevant control plots. 
Secondly, the reference plot approach fails to describe within-burn heterogeneity as it uses mean values per fire plot. To solve these problems, Lhermitte et al. (2010) proposed a pixelbased control plot selection method which follows the same reasoning with respect to the minimization of phenological effects by comparison with image-based control plots. The difference with the reference plot procedure, however, is situated in the fact that the pixelbased method assigns a unique unburned control pixel to each burned pixel. This control pixel selection is based on the similarity between the time series of the burned pixel and the time series of its surrounding unburned pixels for a pre-fire year (Lhermitte et al. 2010). The method allows the quantification of the heterogeneity within a fire plot since each fire pixel is considered independently as a focal study pixel and a control pixel is selected from a contextual neighbourhood around the focal pixel. This approach has been used in studies assessing the temporal dimension of fire impact and subsequent recovery (Veraverbeke et al. 2010b; Lhermitte et al. 2011; Veraverbeke et al. 2011a; Veraverbeke et al. 2012c). Figure 2 presents an example of the principle of the control plot selection procedure on a NDVI time series. With the exception of Veraverbeke et al. (2012c) who also incorporated Land Surface Temperature (LST) and albedo data in the procedure, so far, the control plot selection procedure has only been applied on NDVI data. Nevertheless, in theory, the control plot selection procedure allows any kind of remotely sensed data as input. Moreover, the procedure has the potential to provide valuable reference information other disturbance in which external forces abruptly remove the vegetation (e.g. volcanic eruptions, landslides, hurricanes, tsunamis, etc.).

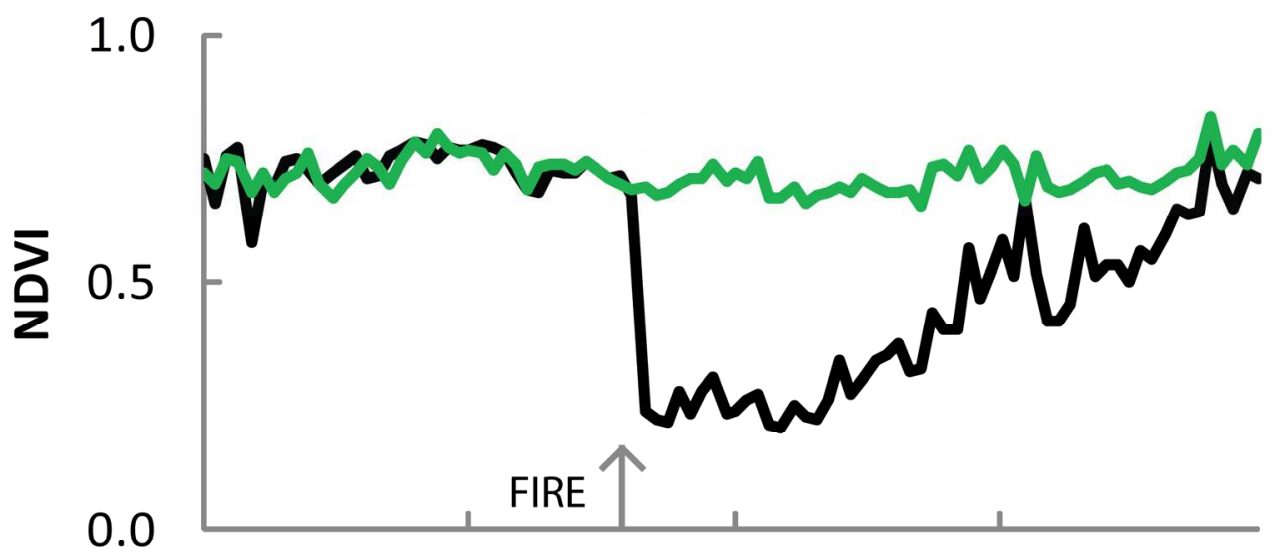

\section{1-Jan-06 1-Jan-07 1-Jan-08 1-Jan-09}

Fig. 2. Example of Normalized Difference Vegetation Index (NDVI) time series of a burned pixel (black line) and its corresponding control pixel (green line). The control pixel mimics how the burned pixel would have behaved without fire occurrence (after Veraverbeke et al. (2012c)). 
Despite of the merits of the control pixel selection procedure as presented by Lhermitte et al. (2010) and Veraverbeke et al. (2010b) two constraints remain. Firstly, due to the necessity to search in larger windows for pixels in the middle of the burn the performance of the procedure is likely to be better near the contours of the burn perimeter. On one hand this is inevitable as the potentially most similar neighbour pixels are burned. On the other hand one could argue that this phenomenon incites to make the control pixel selection settings dependent on the distance to the fire perimeter. The procedure is also affected by a second constraint, i.e. the heterogeneity of the unburned landscape matrix. It is obvious that the procedure will be more optimal in highly homogeneous landscapes, even for large search windows. In contrast, in highly heterogeneous mixtures of different land cover types the procedure will potentially fail to retrieve similar pixels for small window sizes. It is a hard task to uncouple and quantify the effects of both constraints. Solutions to this have the potential to further improve the selection procedure.

\subsubsection{Post-fire albedo and land surface temperature}

Besides the use of optical data and its derivatives (e.g. VIs) some authors focused on the recovery of remotely sensed bioclimatic variables such as albedo and Land Surface Temperature (LST) (Amiro et al. 1999; Lyons and Randerson 2008; Veraverbeke et al. (2012c)). The increase in post-fire LST progressively weakens over time (Veraverbeke et al. (2012c)), whereas Lyons et al. (2008) and Veraverbeke et al. (2012c)) observed that albedo quickly recovers after an initial post-fire drop. The albedo even exceeds pre-fire values when char materials are removed and vegetation starts to regenerate (Veraverbeke et al. (2012c)). Thus, where the immediate fire effect results in an increased absorption of radiative energy, the long-term effect generally is an increased albedo (Amiro et al. 2006; Randerson et al. 2006). The quantification of these effects, together with an accurate estimation of the amount of greenhouse gasses emitted by the fire and the subsequent post-fire carbon sequestration of regenerating vegetation, are necessary for a holistic comprehension of the effect of wildfires on regional and global climate. In this context, Randerson et al. (2006) comprehensively demonstrated that, although the first post-fire year resulted in a net warming, the long-term balance was negative. As such they concluded that an increasing fire activity in the boreal region would not necessarily lead to a net climate warming. Remotely sensed proxies of albedo and LST can also be used to estimate the spatio-temporal behaviour of several radiative budget parameters of paramount biophysical importance such as sensible and latent heat fluxes (Bastiaanssen et al. 1998; Roerink et al. 2000). The immediate post-fire surface warming and its ecological consequences as well as the longterm post-fire temporal development of heat fluxes could form a relatively unexplored and captivating research topic.

\subsubsection{Post-fire monitoring using SAR}

Synthetic-aperture radar (SAR) data has been extensively used for various ecological processes (Kasischke et al. 1997) and have been especially useful in areas characterized by frequent cloud conditions such as the tropics and in the remote locations of the boreal forests. However, the application of SAR data in monitoring vegetation regrowth has been rather limited, while in most of the studies empirical relationships between field measurements and the backscatter values have been investigated. 
Ramsey et al (1999) investigated the use of multiple-polarization aircraft L-band to monitor burn recovery in a coastal marsh. The authors found a significant relationship between $\mathrm{VH}-$ polarization and time-since-burn. In addition, Ramsey et al 1999 examined the same relationship with scaled SAR returns. Scaled by control data [e.g. VH(burn)-VH(control)], all three polarizations $(\mathrm{VV}, \mathrm{HH}, \mathrm{VH})$ regressions were found significant, with $83 \%$ of the timesince-burn explained by the $\mathrm{VH}$ variable.

Kasischke et all (2011) investigated the utility of L-band ALOS PALSAR data $(23.6 \mathrm{~cm}$ wavelength) for estimating low aboveground biomass in a fire-distributed black spruce forests in interior Alaska nearly 20 years after the fire events. Field measurements were analyzed against the radar backscatter coefficient. Significant linear correlations were found between the log of the aboveground biomass and $\sigma^{\circ}(\mathrm{L}-\mathrm{HH})$ and $\sigma^{\circ}$ (L-HV), with the highest correlation found when soil moisture was high. Kasischke et all 2011 concluded that using spaceborne SAR systems to monitor forest regrowth will not only require collection of biomass data to establish the relationship between biomass and backscatter, but may also require developing methods to account for variations in soil moisture.

Kasischke et al (2007) based on the findings of Bourgeau-Chavez et al (2007) explored the relations between soil moisture patterns and post-fire tree recruitment in fire-disturbed black spruce forests in Interior Alaska using ERS-1 and ERS-2 C band (5.7 cm wavelength). Both Kasischke et al (2007) and Bourgeau-Chavez et al (2007) found high correlations between ERS SAR backscatter and measured soil moisture in the burned areas. Furthermore, Kasischke et al (2007) found that the measured levels of tree recruitment are related to the levels of soil moisture: aspen seedlings were able to germinate and grow within the severely burned areas because of adequate soil moisture was present during the growing season. In contrast, low aspen recruitment at a severely burned area was attributed to lower soil moisture.

Minchella et al. (2009) used multitemporal ERS SAR images to monitor the vegetation recovery in a Mediterranean burned area. Following a qualitative approach (analysis of the multitemporal backscattering signatures) they observed that, due to the increase in soil moisture in the backscattering, the measurements, taken throughout at least one year, of the similarity between the backscattering of the burned area and the backscattering of a bare soil around or inside the burned area, may provide a SAR-based index for the vegetation recovery in the burned area. In addition, Minchella et all 2009 used a microwave scattering model. A minimization of the distance between simulated results and measured data has been carried out using the re-growth rate as the key variable. Results showed that the retrieved values were in agreement with in-situ measurements.

Tanase et al 2011 analyzed SAR metrics from burned forested areas in Spain and Alaska. SAR dataset that were used consisted of ERS ( $C$ band), TerraSAR-X (X-band), Environmental Satellite (Envisat) Advanced SAR (ASAR) (C-band) and ALOS PALSAR (Lband) images. The authors concluded that for Mediterranean forests, the L-band HVpolarized SAR backscatter allowed the best differentiation of regrowth phases whereas at Xand C-band the HV-polarized backscatter was less sensitive to modification in forest structure due to the rapid saturation of the signal. For boreal forest four different regrowth phases were separated. Co-polarized repeat-pass coherence presented weak sensitivity to the different forest regrowth phases. Separation was possible only for the most recently affected sites ( $<15$ year since disturbance) regardless of the radar frequency. 


\section{Advances in remote sensing of post-fire monitoring}

Given that older generation sensors have many known limitations with respect to their suitability for studying complex biophysical characteristics (De Jong et al. 2000, Steininger 2000, Sampson et al. 2001, Salas et al. 2002), the need to benefit from new generation of high spatial and spectral resolution sensors as well as active sensors is of critical importance. Accurate quantification of vegetation regeneration could be essential for biodiversity assessment, land cover characterization and biomass modelling (Blackburn and Milton 1995). High spectral resolution facilitates the identification of features while high spatial resolution permits accurate location of features (Gross and Scott 1998). Additionally, advanced multispectral sensors also allow significantly improved signal to noise ratios (Levesque and King 2003).

The development of new hyperspectral remote sensing instruments, both airborne and spaceborne, has provided an opportunity to study vegetation recovery after wildfire (Riaño et al. 2002). A number of recent studies have indicated the advantages of using discrete narrowband data from specific portions of the spectrum, rather than broadband data, to obtain the most sensitive quantitative or qualitative information on vegetation characteristics.

Mitri and Gitas (2010) mapped post-fire vegetation recovery using EO-1 Hyperion imagery and OBIA and an overall accuracy of $75.81 \%$ was reported (Figure 3). Object-oriented image analysis has been developed to overcome the limitations and weaknesses of traditional image processing methods for feature extraction from high resolution images (Mitri and Gitas 2004; Mitri and Gitas 2010). The basic difference, especially when compared with pixel-based procedures is that image object analysis does not classify single pixels but rather image objects that have been extracted in a previous image segmentation step (Baatz and Schape 1999). The concept here is that the information that is necessary to interpret an image is not represented in a single pixel, but in image objects. Object-based classification involves three main steps, namely, image segmentation, object training, and object classification. "Ground truth" information using field spectroradiometry instruments is equally important for validation of representative image wavebands to be used in object-based classification. According to Wicks et al. (2002), object-based classification may result in an increased accuracy and more realistic presentation of the environment.

Furthermore, many applications of remote sensing require high spatial resolution data for a correct determination of small objects. For instance, high spatial resolution imagery can be used before, during, and after a fire to measure fuel potential, access, progress, extent, as well as damage and financial loss. High spatial resolution multispectral data such as QuickBird (60 centimetres in panchromatic and $2.4 \mathrm{~m}$ in multispectral) can identify not only individual tree crowns, but often also the type of tree, estimate biomass, condition and age class (Wang et al. 2004, Palandjian et al. 2009).

LIDAR data have been used extensively for estimating various forest attributes such as canopy height, biomass, basal area and LAI forest variables as diameter at breast height, volume and density (Dubayah and Drake 2000; Lim et al. 2003a; Bortolot and Wynne 2005) and individual tree heights. The limited use of LIDAR data for monitoring vegetation regrowth can be attributed to the limited existence of spaceborne LIDAR Also, the operation of airborne LIDAR can be hampered by weather conditions, it is cost prohibited and can cover only limited areas. 

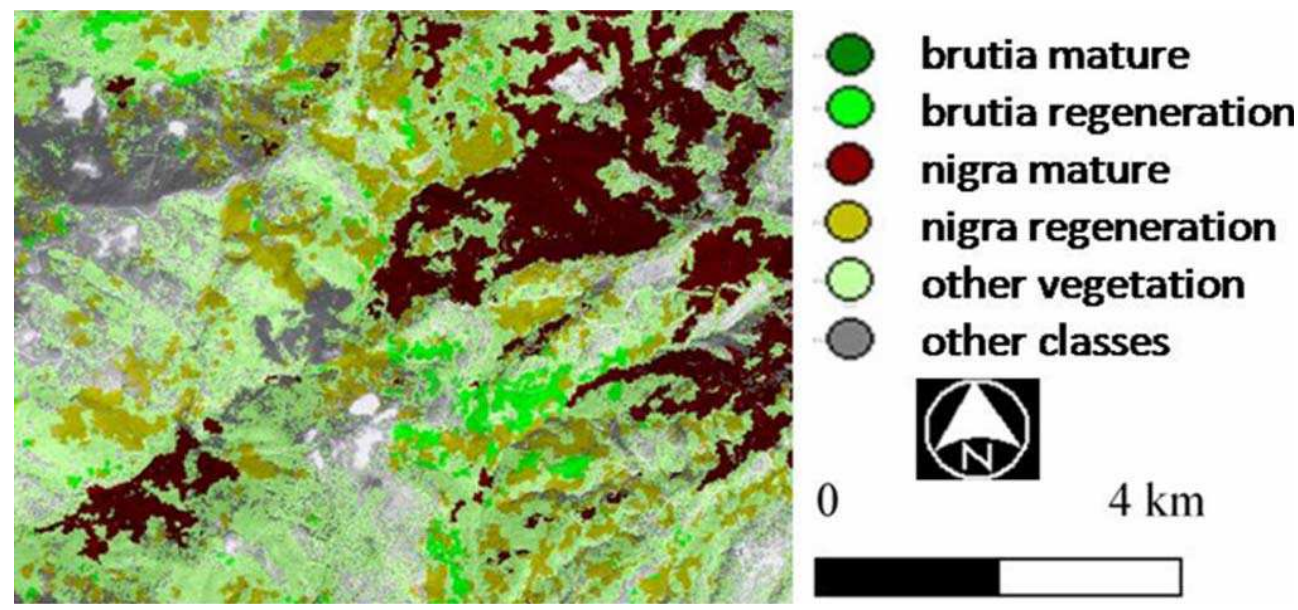

Fig. 3. Example of post-fire recovery map obtained after applying object-based classification in a Mediterranean ecosystem (Mitri and Gitas 2010).

However, it is worth mentioning the work of Wulder et al (2009) who used the integration of Landsat TM/ETM+ imagery and profiling LIDAR transects to characterize post-fire conditions on boreal forest in Canada shortly after the fire event. The main goals of their research were to evaluate whether LIDAR can be used to detect changes in vertical forest structural characteristics associated with the wildfire and find relationships between the vertical information extracted from the LIDAR datasets and horizontal information such as the indices NBR, dNBR and RdNBR extracted from the Landsat datasets in pre- and postfire conditions. The authors had available LIDAR data collected along the same transect preand post-fire and could identify differences in forest structure before and after the fire, but those differences in structure were more related to post fire effects in dense forest than open or sparse forests. However, no significant correlation was found between the Landsat measures of post-fire effect and the LIDAR -derived measures of pre-fire forest structure. Kim et al (2009) used LIDAR intensity values in conjunction with field measurements to distinguish between live and dead standing tree biomass in a mixed coniferous forest in USA. Result of their regression analysis showed that low intensity returns from LIDAR were associated with dead tree biomass. It was suggested that knowing the background value of dead biomass in the forest (from field observations) one can estimate the additional contribution of dead standing tree biomass associated with a fire.

In addition to the airborne LIDAR applications, there has been a study using the satellitebased LIDAR instrument Geoscience Laser Altimeter System (GLAS) onboard ICESAT. Goetz et al (2010) used the LIDAR data acquired from GLAS to derive canopy structure information in burned areas and associated forest regrowth across Alaska. The LIDAR data were stratified by age class (using fire perimeters) and vegetation type (using the EVI calculated from MODIS NBAR). Data were also analyzed in relation to the burn severity. Tree height estimates derived from GLAS data were then compared to field measurements. The results showed that the GLAS data have the utility for inferring height properties of vegetation in fire disturbed areas of boreal forest, but it was mentioned that the data require both careful screening and some knowledge of the study area. 
Furthermore, even though Polarimetric Interferometric SAR (PolInSAR) data has not been used so far for monitoring vegetation regrowth in fire affected areas, PolInSAR proved capable in estimating tree height (Papathanasiou and Cloude, 2001). The height estimation with PolInSAR has been successfully demonstrated on various forest types, ranging from boreal to tropical forests, and achieves high accuracies, (Praks et al. 2007; Garestier et al. 2008; Hajnsek et al. 2009). The operation of single-pass interferometric systems (TanDEM- $X$ has been operating since May 2010) in space opens the door to a new set of unique applications (Krieger et al. 2010), one of them being the monitoring of forest biomass after fire.

\section{Future investigation}

Given that older generation sensors have many known limitations with respect to their suitability for studying complex biophysical characteristics, the need to benefit from new generation of high spatial and spectral resolution sensors as well as active sensors is of critical importance. The development of new remote sensing instruments, both airborne and spaceborne, has provided an opportunity to advance studies and researches on vegetation recovery after wildfire. Future research related to advances in remote sensing in post-fire monitoring is expected to focus on the following:

- Ecosystems with high environmental importance such as tropical forest and savannah ecosystems urgently require a knowledge gain.

- The need to conduct research on the remote sensing of post-fire vegetation recovery supported by field campaigns.

- The potential of spectral indices with a SWIR or MIR spectral band.

- Given that older generation sensors have many known limitations with respect to their suitability for studying complex biophysical characteristics, the need to benefit from new generation of high spatial and spectral resolution sensors and active sensors with different characteristics is of critical importance. Also the use of combined data acquired from more than one sensor.

- New research pathways to study the small-scale spatial patterns of post-fire vegetation recovery are required (e.g. the need to explore the potential of high resolution imagery such as QuickBird and IKONOS to classify individual plants).

- Investigation of the potential of Polarimetric Interferometric SAR data (TanDEM-X) to estimate post-fire biomass.

- Exploitation of advanced image analysis techniques in order to develop automated and transferable procedures.

\section{Conclusions}

Based on a review of the literature, a number of conclusions can be drawn:

- The role of remote sensing is increasingly becoming very important in post-fire monitoring.

- Most research has so far been carried out in areas covered by Mediterranean forests and shrublands.

- Current research is mainly based on the employment of Landsat, MODIS, VGT and AVHRR data. The extensive use of moderate and low resolution imagery is related to time series analysis. 
- Vegetation indices and SMA are the main techniques employed so far in post-fire monitoring while the NDVI is the most commonly used index due to its strong relationship with above-ground biomass in a wide range of ecosystems. Only a small number of studies employ image classification due to the fact that the spatial resolution of the most commonly used satellite sensors exceeds the size of individual regenerating plants

- A number of developments including: the increase in the number of sensors with different characteristics suitable for post-fire monitoring (e.g. LIDAR, hyperspectral), the improved access to and availability of satellite data and derived products, and the development of new methods and advanced digital image analysis techniques (e.g. OBIA, Control plot selection) are expected to move forward research and establish RS an operational tool for post-fire monitoring.

\section{Acknowledgments}

This publication was financially supported by the Aristotle University of Thessaloniki (AUTh) Research Committee. The review was also supported by the Lebanese National Council for Scientific Research (CNRS) and part of the work was carried out at the Jet Propulsion Laboratory, California Institute of Technology, under a contract with the National Aeronautics and Space Administration.

\section{References}

Alcaraz-Segura, D., Chuvieco, E., Epstein, H., Kasischke, E. and Trishchenko, A., 2010. Debating the greening vs. browning of the North American boreal forest: differences between satellite datasets. Global Change Biology 16: 760-770.

Amanatidis, G., Paliatsos, A., Repapis, C. and Bartzis, J., 1993. Decreasing precipitation trend in the Marathon area, Greece. International Journal of Climatology 13: 191201.

Amiro, B., MacPherson, J. and Desjardins, R., 1999. BOREAS flight measurements of forestfire effects on carbon dioxide and energy fluxes. Agricultural and Forest Meteorology 96: 199-208.

Amiro, B., Orchansky, A., Barr, A., Black, T., Chambers, S., Chapin, F., Goulden, M., Litvak, M., Liu, H., McCaughey, J., McMillan, A. and Randerson, J., 2006. The effect of post-fire stand age on the boreal forest energy balance. Agricultural and Forest Meteorology 140: 41-50.

Arai, K., 2008. Nonlinear mixture model of mixed pixels in remote sensing satellite images based on Monte Carlo simulation. Advances in Space Research 41: 1725-1743.

Arianoutsou, M., 1984. Post-fire successional recovery of a phryganic (East Mediterranean) ecosystem. Acta Oecol. (Oecol. Plant.) 5(19): 287- 394.

Asner, G., 1998. Biophysical and biochemical sources of variability in canopy reflectance. Remote Sensing of Environment 64: 234-253.

Asner, G. and Lobell, D., 2000. A biogeophysical approach for automated SWIR unmixing of soils and vegetation. Remote Sensing of Environment 74: 99-112.

Atkinson, P., Cutler, M. and Lewis, H., 1997. Mapping sub-pixel proportional land-cover with AVHRR imagery. International Journal of Remote Sensing 18: 917-935.

Attema, E. P. W. and Ulaby, F. T., 1978. Vegetation modeled as a water cloud. Radio Science 13(2): 357-364. 
Baatz, M. and Schape, A., 1999. Object-Oriented and Multi-Scale Image Analysis in Semantic Networks. In: 2nd International Symposium on Operationalization of Remote Sensing: $15-20$.

Baeza, M. and Roy, J., 2008. Germination of an obligate seeder (Ulex parviflorus) and consequences for wildfire management. Forest ecology management 256: 685-693.

Bailey, J. and Covington, W., 2002. Evaluating ponderosa pine regeneration rates following ecological restoration treatments in northern Arizona, USA. Forest ecology and management 155.

Baret, F. and Guyot, G., 1991. Potentials and limits of vegetation indices for LAI and APAR assessment. Remote Sensing of Environment 35: 161-173.

Bastiaanssen, W., Menenti, M., Feddes, R. and Holtslag, A., 1998. A remote sensing surface energy balance algorithm for land (SEBAL) 1. Formulation. Journal of Hydrology 212-213: 198-212.

Beaty, R. and Taylor, A., 2001. Spatial and temporal variation of fire regimes in a mixed conifer forest landscape, southern Cascades, California, USA. Journal of Biogeography 28: 955-966.

Belda, F. and Meliá, J., 2000. Relationships between climatic parameters and forest vegetation: application to burned area in Alicante (Spain) Forest ecology and management 135 195-204.

Bertolette, D. and Spotskey, D., 2001. Remotely sensed burn severity mapping. In: H. David Harmon, Michigan, Crossing Boundaries in Park Management: Proceedings of the 11th Conference on research and Resource Management in Parks and on Public Lands, The George Wright Society: 44-51.

Bisson, M., Fornaciai, A., Coli, A., Mazzarini, F. and Pareschi, M., 2008. The Vegetation Resilience After Fire (VRAF) index: development, implementation and an illustration from central Italy. International Journal of Applied Earth Observation and Geoinformation 10: 312-329.

Bobbe, T., Lachowski, H., Maus, P., Greer, J. and Dull, C., 2001. A primer on mapping vegetation using remote sensing. International Journal of Wildland Fire 10: 277287.

Bonham, C., 1989. Measurements for terrestrial vegetation. Wiley, New York, NY, USA.

Bortolot, Z. J. and Wynne, R. H., 2005. Estimating forest biomass using small footprint LIDAR data: An individual tree-based approach that incorporates training data. ISPRS Journal of Photogrammetry \& Remote Sensing 59(6): 342- 360.

Bourgeau-Chavez, L. L., Kasischke, E. S., Riordan, K., Brunzell, S., Nolan, M., Hyer, E., Slawski, J., Medvecz, M., Walters, T. and Ames, S., 2007. Remote monitoring of spatial and temporal surface soil moisture in fire disturbed boreal forest ecosystems with ERS SAR imagery. International Journal of Remote Sensing 28(10): 2133 2162.

Brewer, C. K., Winne, J. C., Redmond, R. L., Opitz, D. W., Mangrich, M. V., 2005. Classifying and mapping wildfire severity: A comparison of methods. Photogrammetric Engineering and Remote Sensing 71: 1311-1320.

Broge, N. and Leblanc, E., 2000. Comparing prediction power and stability of broadband and hyperspectral vegetation indices for estimation of green leaf area index and canopy chlorophyll density. Remote Sensing of Environment 76: 156-172.

Buhk, C., Meyn, A. and Jentsch, A., 2007. The challenge if plant regeneration after fire in the Mediterranean Basin: scientific gaps in our knowledge on plant strategies and evolution of traits. Plant ecology 192: 1-19. 
Calvo, L., Tarrega, R. and Luis, E., 1991. Regeneration in Quercus Pyrenaica Ecosystems After Surface Fires. International Journal of Wildland Fire 1: 205-210.

Calvo, L., Tarrega, R. and Luis, E., 1994. Comparative study of post-fire successional development in two communities with different maturity stage in Quercus pyrenaica climax. In: Viegas, D.X. (Ed.) Proceedings of the 2nd International Conference on Forest Fire Research : Coimbra: 951-960.

Calvo, L., Tarrega, R. and Luis, E., 1998. Twelve years of vegetation changes after fire in an Erica australis community. In: Trabaud, L. (Ed.) Fire Management and Landscape ecology. Fairfield, Whashington, International Association of Wildland Fire: 123136.

Calvo, L., Tarrega, R. and Luis, E., 2002. The dynamics of Mediterranean shrubs species over 12 years following perturbations. Plant ecology 160: 25-42.

Carlson, T. and Ripley, T., 1997. On the relation between NDVI, fractional vegetation cover and leaf area index. Remote Sensing of Environment 62: 241-252.

Carranza, M., Ricotta, C., Napolitano, P., Massaro, E. and Blasi, C., 2001. Quantifying postfire regrowth of remotely sensed Mediterranean vegetation with percolation-based methods. Plant Biosystems 135: 311-318.

Carreiras, J., Pereira, M. C. J. and Pereira, S. J., 2006. Estimation of tree canopy cover in evergreen oak woodlands using remote sensing. Forest Ecology and Management 23: 45-53.

Caturla, N., Raventós, J., Guàrdia, R. and Vallejo, R., 2000. Early post-fire regeneration dynamics of Brachypodium retusum Pers. (Beauv.) In old fields of the Valencia region (eastern Spain). Acta Oecologica 21: 1-12.

Chuvieco, E. and Congalton, R., 1988. Mapping and inventory of forest fires from digital processing of TM data. Geocarto International 4: 41-53.

Clemente, A., Rego, F. and Correia, A., 1996. Demographic patterns and productivity of post-fire regeneration in Portuguese Mediterranean maquis. Int. J. of Wildland Fire 6(1): 5-12.

Clemente, R., Navarro Cerrillo, R. and Gitas, I., 2009. Monitoring post-fire regeneration in Mediterranean ecosystems by employing multitemporal satellite imagery. International Journal of Wildland Fire 18: 648-658.

Cruz, A., Pérez, B. and Moreno, J. M., 2003. Resprouting of the Mediterranean type shrub Erica australis with modified lignotuber carbohydrate content. J. Ecol 91: 348-356.

Cuevas-Gonzalez, M., Gerard, F., Baltzer, H. and Riano, D., 2008. Studying the change in fAPAR after forest fire in Siberia using MODIS. International Journal of Remote Sensing 29: 6873-6892.

Cuevas-Gonzalez, M., Gerard, F., Baltzer, H. and Riano, D., 2009. Analysing forest recovery after wildfire disturbance in boreal Siberia using remotely sensed vegetation indices. Global Change Biology 15: 561-577.

Dafis, S., 1990. Prevention of fire and postfire rehabilitation using silvicultural methods. In: Workshop on forest fires in Greece. Department of Forestry and Natural Environment, Aristotelian University of Thessaloniki, Thessaloniki, Greece. 65-72.

Daskalakou, E. and Thanos, C., 1997. Post-fire establishment and survival of Aleppo pine seedlings. In: P. Balabanis, G. Eftichidis, R. Fantechi (Eds.) Forest fire risk and management European Commission, Directorate General XII, Science, Research and Development, Luxembourg. 357-368. 
Daskalakou, E. N. and Thanos, C. A., 1996. Aleppo Pine (Pinus Halepensis) Postfire Regeneration: the Role of Canopy and Soil Seed Banks. International Journal of Wildland Fire 6: 59-66.

Daskalakou, E. N. and Thanos, C. A., 2004. Postfire regeneration of Aleppo pine - The temporal pattern of seedling recruitment. Plant ecology 171: 81-89.

De Luis, M., Garcia-Cano, M., Cortina, J., Raventos, J., Gonzales-Hidalgo, J. and Sanchez, J., 2001. Climatic trends, disturbances and short-term vegetation dynamics in a Mediterranean shrubland. Forest ecology and management: 25-37.

Diaz-Delgado, R., Lloret, F. and Pons, X., 2003. Influence of fire severity on plant regeneration by means of remote sensing. International Journal of Remote Sensing 24: 1751-1763.

Diaz-Delgado, R., Lloret, F., Pons, X. and Terradas, J., 2002. Satellite evidence of decreasing resilience in mediterranean plant communities after recurrent wildfires. Ecology 83: 2293-2303.

Díaz-Delgado, R. and Pons, X., 2001. Spatial patterns of forest fires in Catalonia (NE of Spain) along the period 1975-1995. Analysis of vegetation recovery after fire. Forest ecology and management 147: 67-74.

Drake, N., Mackin, S. and Settle, J., 1999. Mapping vegetation, soils and geology in semiarid shrublands using spectral matching and mixture modeling of SWIR AVIRIS imagery. Remote Sensing of Environment 68: 12-25.

Dubayah, R. O. and Drake, J. B., 2000. LIDAR remote sensing for forestry applications. Journal of Forestry 98: 44-46.

Elmore, A., Mustard, J., Manning, S. and Lobell, D., 2000. Quantifying vegetation change in semiarid environments: precision and accuracy of spectral mixture analysis and the normalized difference vegetation index. Remote Sensing of Environment 73: 87-102.

EPA, 2001. Inventory of U.S. Greenhouse Gas Emissions and Sinks: 1990 - 1999. In: Environmental Protection Agency, U.S. 75-85.

Escuin, S., Fernández-Rebollo, P. and Navarro, R. M., 2002. Aplicación de escenas Landsat a la asignación de grados de afectación producidos por incendios forestales. Revista de Teledetección 1: 25-36.

Eshel, A., Henig-Sever, N. and Ne'eman, G., 2000. Spatial variation of seedling distribution in an east Mediterranean pine woodland at the beginning of post-fire succession. Plant Ecology 148: 175-182.

Ferrandis, P., Herranz, J. M. and Martínez-Sánchez, J. J., 1996. The Role of Soil Seed Bank in the Early Stages of Plant Recovery After Fire in a Pinus Pinaster Forest in SE Spain. International Journal of Wildland Fire 6: 31-35.

García-Ruiz, J., Lasanta, T., Ruiz-Flano, P., Ortigosa, L., White, S., González, C. and Martí, C., 1996. Land-use changes and sustainable development in mountain areas: A case study in the Spanish Pyrenees. Landscape Ecology 11: 267-277.

Garestier, F., Dubois Fernandez, P. and Papathanassiou, K., 2008. Pine forest height inversion using single-pass X-band PolInSAR data. IEEE Trans. Geosci. Remote Sensing 46: 59-68.

Gitas, I., 1999. Geographical Information Systems and Remote Sensing in mapping and monitoring fire-altered forest landscapes. PhD dissertation. Department of Geography, University of Cambridge: 237.

Gitas, I., De Santis, A. and Mitri, G., 2009. Remote sensing of burn severity. In: Chuvieco, E.,. (Eds), Earth observation of wildland fires in mediterranean ecosystems. SpringerVerlag, Berlin: 129-148. 
Goetz, S., Fiske, G. and Bunn, A., 2006. Using satellite time-series data sets to analyze fire disturbance and forest recovery across Canada. Remote Sensing of Environment 101: 352-365.

Goetz, S., Sun, M., Baccini, A. and Beck, P., 2010. Synergetic use of spaceborne LIDAR and optical imagery for assessing forest fire disturbance: an Alaskan case study. Journal of Geophysical Research 115(G00E07).

Gould, K., Fredericksen, T., Morales, F., Kennard, D., Putz, F., Mostacedo, B. and Toledo, M., 2002. Post-fire tree regeneration in lowland Bolivia: implications for fire management. Forest ecology and management 165: 225-234.

Gouveia, C., DaCamara, C. and Trigo, R., 2010. Post-fire vegetation recovery in Portugal based on spot/vegetation data. Natural Hazards and Earth System Sciences 10: 673684.

Hajnsek, I., Kugler, F., Lee, S. and Papathanassiou, K., 2009. Tropical-forest-parameter estimation by means of Pol-InSAR: The INDREX-II campaign. IEEE Trans. Geosci. Remote Sensing 47: 481-493.

Hall, F., Botkin, D., Strebel, D., Woods, K. and Goetz, S., 1991. Large-scale patterns of forest succession as determined by remote sensing. Ecology 72: 628-640.

Henry, M. and Hope, S., 1998. Monitoring post-burn recovery of chaparral vegetation in southern California using multi-temporal satellite data. International Journal of Remote Sensing 19: 3097-3107.

Hernandez-Clemente, R., Navarro, R., Hernandez-Bermejo, J., Escuin, S. and Kasimis, N., 2009. Analysis of postfire vegetation dynamics of Mediterranean shrub species based on terrestrial and NDVI data. Environmental Management 43: 876-887.

Herranz, J., Ferrandis, P. and Martínez-Sánchez, J., 1999. Influence of heat on seed germination of nine woody Cistaceae species. International Journal of Wildland Fire 9: 173-182.

Hicke, J., Asner, G., Kasischke, E., French, N., Randerson, J., Collatz, J., Stocks, B., Tucker, C., Los, S. and Field, C., 2003. Postfire response of North American boreal forest net primary productivity analyzed with satellite observations. Global Change Biology 9: 1145-1157.

Hope, A., Tague, C. and Clark, R., 2007. Characterizing post-fire vegetation recovery of California chaparral using TM/ETM+ time-series data. International Journal of Remote Sensing 28: 1339-1354.

Huete, A., 1988. A Soil-Adjusted Vegetation Index (SAVI). Remote Sensing of Environment 25: 295-309.

Hunter, M. E., Omi, P. N., Martinson, E. J. and Chong, G. W., 2006. Establishment of nonnative plant species after wildfires: effects of fuel treatments, abiotic and biotic factors, and post-fire grass seeding treatments. International Journal of Wildland Fire 15: 271-281.

Idris, M., Kuraji, K. and Suzuki, M., 2005. Evaluating vegetation recovery following largescale forest fires in Borneo and northeastern China using multi-temporal NOAA/AVHRR images. Journal of Forest Research 10: 101-111.

Inbar, M., Tamir, M. and Wittenberg, L., 1998. Runoff and erosion processes after a forest fire in Mount Carmel, a Mediterranean area. Geomorphology 24: 17-33.

Jabukauskas, M., Lulla, K. and Mausel, P., 1990. Assessment of vegetation change in a firealtered forest landscape. Photogrammetric Engineering and Remote Sensing 56: 371-377. 
Jacobson, C., 2010. Use of linguistic estimates and vegetation indices to assess post-fire vegetation regrowth in woodland areas. International Journal of Wildland Fire 19: 94-103.

Jakubauskas, M., 1988. Postfire vegetation change detection using LANDSAT MSS and TM data. School of Graduate Studies. Terre Haute, Indiana, Indiana State University: 99.

Jakubauskas, M., Lulla, K. and Mausel, P., 1990. Assessment of vegetation change in a firealtered forest landscape. Photogrammetric Engineering and Remote Sensing 56: 371-377.

Ju, J. and Roy, D., 2008. The availability of cloud-free Landsat ETM+ data over the conterminous United States and globally. Remote Sensing of Environment 112: 1196-1211.

Kasischke, E. S., Bourgeau-Chavez, L. L. and Johnstone, J. F., 2007. Assessing spatial and temporal variations in surface soil moisture in fire-disturbed black spruce forests in Interior Alaska using spaceborne synthetic aperture radar imagery-Implications for post-fire tree recruitment. Remote Sensing of Environment 108: 42-58.

Kasischke, E. S., Melack, J. M. and Dobson, M. C., 1997. The use of imaging radars for ecological applications-A review. Remote Sensing Environment 59: 141-156.

Kasischke, E. S., Tanase, M. A., Bourgeau-Chavez, L. L. and Borr, M., 2011. Soil moisture limitations on monitoring boreal forest regrowth using spaceborne L-band SAR data. Remote Sensing of Environment 115: 227-232.

Kavgaci, A., Carni, A., Basaran, S., Basaran, M. A., Kosir, P., Marinsek, A. and Silc, U., 2010. Long-term post-fire succession of Pinus brutia forest in the east Mediterranean. International Journal of Wildland Fire 19: 599-605.

Kazanis, D. and Arianoutsou, M., 2004. Long-term post-fire vegetation dynamics in Pinus halepensis forests of central Greece: a functional-group approach. Plant ecology 171: 101-121.

Keeley, J., 2000. Chaparral. In: M. Barbour and W. Billings, North American terrestrial vegetation, New York, Cambridge University Press: 204-253.

Kennard, D., Gould, K., Putz, F., Fredericksen, T. and Morales, F., 2002. Effect of disturbance intensity on regeneration meachanisms in a tropical dry forest. Forest ecology and management 162: 197-208.

Kim, Y., Yang, Z., Cohen, W. B., Pflugmacher, D., Lauver, C. L. and Vankat, J. L., 2009. Distinguishing between live and dead standing tree biomass on the North Rim of Grand Canyon National Park, USA using small-footprint LIDAR data. Remote Sensing of Environment 113: 2499-2510.

Kozlowski, T., 2002. Physiological ecology of natural regeneration of harvested and disturbed forest stands: implications for forest management. Forest ecology and management: 195-221.

Krieger, G., Papathanassiou, K. P., Younis, M., Moreira, A. and Hajnsek, I., 2010. Interferometric Synthetic Aperture Radar (SAR) Missions Employing Formation Flying A German Earth satellite system is designed to produce data on forest structure, biomass, tectonic shifts and glacier movements, and to advance understanding of Earth dynamics. Proceedings of the IEEE 98(5).

Le Houerou, H., 1987. Vegetation wildfires in the Mediterranean basin: evolution and trends. Ecologia Mediterranea 13: 13-24.

Lhermitte, S., Verbesselt, J., Verstraeten, W. and Coppin, P., 2010. A pixel based regeneration index using time series similarity and spatial context. Photogrammetric Engineering and Remote Sensing 76: 673-682. 
Lhermitte, S., Verbesselt, J., Verstraeten, W. W., Veraverbeke, S. and Coppin, P., 2011. Assessing intra-annual vegetation regrowth after fire using the pixel based regeneration index. ISPRS Journal of Photogrammetry and Remote Sensing 66: 17-27.

Li, M., Qu, J. and Hao, X., 2008. Detecting vegetation change with satellite remote sensing over 2007 Georgia wildfire regions. Journal of Applied Remote Sensing 2(021505).

Lim, K., Treitz, P., Wulder, M., Stonge, B. and Flood, M., 2003a. LIDAR remote sensing of forest structure. Progress in Physical Geography 27(1): 88-106.

Lloret, F. and Vilà, M., 1997. Clearing of vegetation in Mediterranean garrigue: response after a wildfire. Forest ecology and management 93: 227-234.

Lyons, E. J., Y. and Randerson, J., 2008. Changes in surface albedo after fire in boreal forest ecosystems of interior Alaska assessed using MODIS satellite observations. Journal of Geophysical Research 113(G02012).

Maheras, P., 1988. Changes in precipitation conditions in the western Mediterranean over the last century. J. Clim 8: 179-189.

Malak, D. and Pausas, J., 2006. Fire regime and post-fire Normalized Difference Vegetation Index changes in the eastern Iberian peninsula. International Journal of Wildland Fire 15: 407-413.

Marchetti, M., Ricotta, C. and Volpe, F., 1995. A qualitative approach to the mapping of post-fire regrowth in Mediterranean vegetation with Landsat TM data. International Journal of Remote Sensing 16: 2487-2494.

Martínez-Sánchez, J., Ferrandis, P., De las Heras, J. and Herranz, J., 1999. Effect of burnt wood removal on the natural regeneration of Pinus halepensis after fire in a pine forest in Tus valley (SE Spain). Forest ecology and management: 1-10.

McMichael, C., Hope, A., Roberts, D. and Anaya, M., 2004. Post-fire recovery of leaf area index in California chaparral: a remote sensing-chronosequence approach. International Journal of Remote Sensing 25: 4743-4760.

Miller, M., 2000. Fire autoecology. In: J.K. Brown and J.K. Smith (Eds.), Wildland Fire in Ecosystems. Ogden, Uta: RMRS-GTR-42, USDA Forest Service, Rocky Mountain Research Station.

Minchella, A., Del Frate, F., Capogna, F., Anselmi, S. and Manes, F., 2009. Use of multitemporal SAR data for monitoring vegetation recovery of Mediterranean burned areas. Remote Sensing of Environment 113: 588-597.

Mitchell, M. and Yuan, F., 2010. Assessing forest fire and vegetation recovery in the Black Hills, South Dakota. GIScience and Remote Sensing 47: 276-299.

Mitri, G. and Gitas, I., 2004. A semi-automated object-oriented model for burned area mapping in the Mediterranean region using Landsat-TM imagery. International Journal of Wildland Fire 13(3): 367-376.

Mitri, G. and Gitas, I., 2008. Mapping the severity of fire using object-based classification of IKONOS imagery. International Journal of Wildland Fire 17: 431-442.

Mitri, G. and Gitas, I. Z., 2010. Mapping postfire vegetation recovery using EO-1 Hyperion imagery. IEEE Transactions on Geoscience and Remote Sensing 48: 1613-1618.

Mooney, R. and Hobbs, R., 1986. Resilience at the individual plant level. In: B.B. Dell, D. Lamont, and A. Hopkins (Eds.), Resilience in Mediterranean - type ecosystems: 65-82.

Moreno, J. M., Pineda, F. and Rivas-Martinez, S., 1990. Climate vegetation at the Eurosiberian- Mediterranean boundary in the Iberian Peninsula. Journal of Vegetation Science 1: 233-244. 
Mouillet, F., Rambal, S. and Lavorel, S., 2001. A generic process-based SImulator for meditERRanean landscApes (SIERRA): design and validation exercises. Forest ecology and management: 75-97.

Naveh, Z., 1991. The role of fire in Mediterranean vegetation. Botanica Chronika (Greece) 10: 385-405.

Ne'eman, G., Fotheringham, C. J. and Keeley, J., 1999. Patch to landscape patterns in post fire recruitment of a serotinous conifer. Plant ecology 12: 235-242.

Nepstad, D., Verssimo, A., Alencar, A., Nobre, C., Lima, E., Lefebvre, P., Schlesinger, P., Potter, C., Moutinho, P., Mendoza, E., Cochrane, M. and Brooks, V., 1999. Large-scale impoverishment of Amazonian forest by logging and fire. Nature 398: 505-508.

Oliver, C., 1981. Forest development in North America following major disturbances. Forest ecology and management 3: 153-168.

Oliver, C. and Larson, B., 1996. Forest Stand Dynamics. Wiley, New York, NY, USA.

Palandjian, D., Gitas, I. and Wright, R., 2009. Burned area mapping and post-fire impact assessment in the Kassandra peninsula (Greece) using Landsat TM and Quickbird data. Geocarto International 24: 193-205.

Papathanassiou, K. P. and Cloude, S. R., 2001. Single-Baseline Polarimetric SAR Interferometry. IEEE Transactions on Geoscience and Remote Sensing 39(11).

Patterson, M. and Yool, S., 1998. Mapping fire-induced vegetation mortality using Landsat thematic mapper data: A comparison of linear transformation techniques. Remote Sensing of Environment 65: 132-142.

Pausas, J., Carbó, E., Caturla, R., Gil, J. and Vallejo, R., 1999. Post-fire regeneration patterns in the eastern Iberian Peninsula. Acta Oecologica 20: 499-508.

Pausas, J. and Verdu, M., 2005. Plant persistence traits in the fire-prone ecosystems of the Mediterranean basin: a phylogenetic approach. Oikos 109: 196-202.

Pausas, J. G., Ouadah, N., Ferran, A., Gimeno, T. and Vallejo, R., 2003. Fire severity and seedling establishment in Pinus halepensis woodlands, Eastern Iberian Peninsula. Plant Ecology 169: 205- 213.

Perez-Cabello, F., Echeverria, M., Ibarra, P. and De la Riva, J., 2009. Effects of fire on vegetation, soil and hydrogeomorphological behavior in Mediterranean ecosystems. In: Earth Observation of Wildland fires in Mediterranean ecosystems (Emilio Chuvieco Ed.) - Springer: 111-128.

Perula, V. G., Cerrillo, R. N., Reboloo, P. F. and Murillo, G. V., 2003. Postfire regeneration in Pinus pinea L. and Pinus pinaster Aiton in Andalucia (Spain). Env. Manage 31(1): 86-99.

Peterson, S. and Stow, D., 2003. Using multiple image endmember spectral mixture analysis to study chaparral regrowth in Southern California. International Journal of Remote Sensing 24: 4481-4504.

Piñol, J., Terradas, J. and Lloret, F., 1998. Climatic warming hazard, and wildfire occurrence in coastal eastern Spain. Climate Change 38: 345-357.

Praks, J., Kugler, F., Papathanassiou, K., Hajnsek, I. and Hallikainen, M., 2007. Tree height estimation for boreal forest by means of L- and X-band PoLInSAR and HUTSCAT profiling scatterometer. IEEE Geosci. Remote Sens. Lett 4: 466-470.

Qi, J., Chehbounidi, A., Huete, A., Kerr, Y. and Sorooshian, S., 1994. A modified soil adjusted vegetation index. Remote Sensing of Environment 48: 119-126.

Ramsey, E., Nelson, G., Sapkota, S., Laine, S., Verdi, J. and Krasznay, S., 1999. Using multiple-polarization L-band radar to monitor marsh burn recovery. IEEE Transactions on Geoscience and Remote Sensing 37: 636-639. 
Randerson, J., Liu, H., Flanner, M., Chamber, S., Jin, Y., Hess, P., Pfister, G., Mack, M., Treseder, K., Welp, L., Chapin, F., Harden, J., Goulden, M., Lyons, E., Neff, J., Schuur, E. and Zender, C., 2006. The impact of boreal forest fire on climate warming. Science 314: 1130-1132.

Rego, F., 1992. Land use changes and wildfires. In: A. Teller, P. Mathy, J. Jeffers (Eds.) Response of forest fires to environmental change, Elsevier, London: 367-373.

Riaño, D., Chuvieco, E., Ustin, S., Zomer, R., Dennison, P., Roberts, D. and Salas, J., 2002. Assessment of vegetation regeneration after fire through multitemporal analysis of AVIRIS images in the Santa Monica Mountains. Remote Sensing of Environment 79: 60-71.

Ricotta, C., Avena, G., Olsen, E., Ramsey, R. and Winn, D., 1998. Monitoring the landscape stability of Mediterranean vegetation in relation to fire with a fractal algorithm. International Journal of Remote Sensing 19: 871-881.

Roberts, D., Gardner, M., Church, R., Ustin, S., Scheer, G. and Green, R., 1998. Mapping chaparral in the Santa Monica mountains using multiple endmember spectral mixture models. Remote Sensing of Environment 65: 267-279.

Roder, A., Hill, J., Duguy, B., Alloza, J. and Vallejo, R., 2008. Using long time series of Landsat data to monitor fire events and post-fire dynamics and identify driving factors. A case study in the Ayora region (eastern Spain). Remote Sensing of Environment 112: 259-273.

Roerink, G., Su, Z. and Menenti, M., 2000. S-SEBI: A simple remote sensing algorithm to estimate the surface energy balance. Physics and Chemistry of the Earth 25: 147-157.

Rogan, J. and Franklin, J., 2001. Mapping wildfire burn severity in southern California forests and shrublands using Enhanced Thematic Mapper imagery. Geocarto International 16: 1-11.

Rogge, D., Rivard, B., Zhang, J. and Feng, J., 2006. Iterative spectral unmixing for optimizing per-pixel endmember sets. IEEE Transactions on Geoscience and Remote Sensing 44: 3725-3736.

Sankey, T., Moffet, C. and Weber, K., 2008. Postfire recovery of sagebrush communities: assessment using SPOT-5 and very large-scale aerial imagery. Rangeland Ecology and Management 61: 598-604.

Santalla, S., Marcos, E., Valbuena, L., Calvo, L., Tárrega, R. and Luis, E., 2002. First years of regeneration in Quercus pyrenaica forest and Pinus pinaster stand after wildland fire. In: D. Viegas (Ed.) Forest Fire Research \& Wildland Fire Safety, Millpress, Rotterdam.

Schroeder, D. and Perera, A., 2002. A comparison of large-scale spatial vegetation patterns following clearcuts and fires in Ontario's boreal forests. Forest Ecology and Management 159: 217-230.

Segah, H., Tani, H. and Hirano, T., 2010. Detection of fire impact and vegetation recovery over tropical peat swamp forest by satellite data and ground-based NDVI instrument. International Journal of Remote Sensing 31: 5297-5314.

Shaw, D., Malthus, D. and Kupiec, J., 1998. High-spectral resolution data for monitoring Scots pine (Pinus sylvestris L.) regeneration. International Journal of Remote Sensing 19: 2601-2608.

Skordilis, A. and Thanos, C., 1995. Seed stratification and germination strategy in the Mediterranean pines Pinus brutia and P. halepensis. Seed Science Research 5: 151-160. 
Smith, R. and Woodgate, P., 1985. Appraisal of fire damage and inventory for timber salvage by remote sensing in mountain ash forests in Victoria. Australian Journal of Forestry 48(4): 252-263.

Somers, B., Verbesselt, J., Ampe, E., Sims, N., Verstraeten, W. W. and Coppin, P., 2010. Spectral mixture analysis to monitor defoliation in mixed-age Eucalyptus globulus Labill plantations in southern Australia using Landsat 5-TM and EO-A Hyperion data. International Journal of Applied Earth Observation and Geoinformation 12: 270-277.

Steyaert, L., Hall, F. and Loveland, T., 1997. Land cover mapping, fire regeneration, and scaling studies in the Canadian boreal forest with $1 \mathrm{~km}$ AVHRR and Landsat TM data. International Journal of Geophysical Research 102: 29581-29598.

Stueve, K., Cerney, D., Rochefort, R. and Kurt, L., 2009. Post-fire tree establishment patterns at the alpine treeline ecotone: Mount Rainier National Park, Washington, USA. Journal of Vegetation Science 20: 107-120.

Tanase, M., Riva, J.d.l., Santoro, M., Pérez-Cabello, F., Kasischke, E., 2011. Sensitivity of SAR data to post-fire forest regrowth in Mediterranean and boreal forests. Remote Sensing of Environment 115: 2075-2085.

Tarrega, R., Luis-Calabuig, E. and Valbuena, L., 2001. Eleven years of recovery dynamic after experimental burning and cutting in two Cistus communities. Acta Oecologica 22: $277-283$.

Telesca, L. and Lasaponara, R., 2006. Pre- and post-fire behavioral trends revealed in satellite NDVI time series. Geophysical Research Letters 33(L14401).

Thanos, C., Daskalakou, E. and Nikolaidou, S., 1996. Early post-fire regeneration of a Pinus halepensis forest on Mount Parnis, Greece. Journal of Vegetation Science 7: 273-280.

Thanos, C. A. and Georghiou, K., 1988. Ecophysiology of fire-stimulated seed germination in Cistus incanus ssp creticus (L.) Heywood and C. salvifolius L. Plant, Cell and Environment 11: 841-849.

Thenkabail, P., Smith, R. and De-Pauw, E., 2000. Hyperspectral vegetation indices for determining agricultural crop characteristics. Remote Sensing of Environment 71: 158-182.

Thenkabail, P., Smith, R. and De-Pauw, E., 2002. Evaluation of narrowband and broadband vegetation indices for determining optimal hyperspectral wavebands for agricultural crop characterization. Photogrammetric Engineering and Remote Sensing 68: 607-621.

Torn, M. and Fried, J., 1992. Predicting the impacts of global warming on wildland fire. Climate Change 21: 257-274.

Trabaud, L., 1994. Plant and fire variability relationships more specifically in the Mediterranean basin. In: 2nd International Conference in Forest Fire Research, Coimbra, Portugal: 53-58.

Tsitsoni, T., 1997. Conditions determining natural regeneration after wildfires in the Pinus halepensis (Miller, 1768) forests of Kassandra Peninsula (North Greece). Forest ecology and management 92: 199-208.

Tucker, C., 1979. Red and photographic infrared linear combinations for monitoring vegetation. Remote Sensing of Environment 8: 127-150.

van Leeuwen, W., 2008. Monitoring the effects of forest restoration treatments on post-fire vegetation recovery with MODIS multitemporal data. Sensors 8: 2017-2042.

van Leeuwen, W., Casady, G., Neary, D., Bautista, S., Alloza, J., Carmel, J., Wittenberg, L., Malkinson, D. and Orr, B., 2010. Monitoring post-wildfire vegetation response with 
remotely sensed time series data in Spain, USA and Israel. International Journal of Wildland Fire 19: 75-93.

van Wagtendonk, J., Root, R., Key, C. and Running, S., 2004. Comparison of AVIRIS and Landsat ETM+ detection capabilities for burn severity. International Journal of Wildland Fire 92: 397-408.

Veraverbeke, S., Verstraeten, W. W., Lhermitte, S. and Goossens, R., 2010a. Illumination effects on the differenced Normalized Burn Ratio's optimality for assessing fire severity. International Journal of Applied Earth Observation and Geoinformation 12: 60-70.

Veraverbeke, S., Lhermitte, S., Verstraeten, W. W. and Goossens, R., 2010b. The temporal dimension of differenced Normalized Burn Ratio (dNBR) fire/burn severity studies: the case of the large 2007 Peloponnese wildfires in Greece. Remote Sensing of Environment 114: 2548-2563.

Veraverbeke, S., Lhermitte, S., Verstraeten, W. W. and Goossens, R., 2011a. A timeintegrated MODIS burn severity assessment using the multi-temporal differenced Normalized Burn Ratio (dNBRMT). International Journal of Applied Earth Observation and Geoinformation 13: 52-58.

Veraverbeke, S., Somers, B., Gitas, I., Katagis, T., Polychronaki, A. and Goossens, R., 2012a. Spectral mixture analysis to assess post-fire vegetation regeneration using Landsat Thematic mapper imagery: accounting for soil brightness variation. International Journal of Applied Earth Observation and Geoinformation 14: 1-11.

Veraverbeke, S., Gitas, I., Katagis, T., Polychronaki, A., Somers, B. and Goossens, R., 2012b. Assessing post-fire vegetation recovery using red-near infrared vegetation indices: accounting for background and vegetation variability. ISPRS Journal of Photogrammetry and Remote Sensing in press.

Veraverbeke, S., Verstraeten, W. W., Lhermitte, S., Van De Kerchove, R. and Goossens, R., 2012c. Spaceborne assessment of post-fire changes in vegetation, land surface temperature and surface albedo. International Journal of Wildland Fire in press.

Vicente-Serrano, S., Perez-Cabello, F. and Lasanta, T., 2008. Assessment of radiometric correction techniques in analyzing vegetation variability and change using time series of Landsat images. Remote Sensing of Environment 112: 3916-3934.

Viedma, O., Melia, J., Segarra, D. and Garcia-Haro, J., 1997. Modeling rates of ecosystem recovery after fires by using Landsat TM data. Remote Sensing of Environment 61: 383-398.

Vila, G. and Barbosa, P., 2010. Post-fire vegetation regrowth detection in the Deiva Marina region (Liguria-Italy) using Landsat TM and ETM+ data. Ecological Modelling 221: 75-84.

Wahren, C., Papst, W. and Williams, R., 2001. Early post-fire regeneration in subalpine heathland and grassland in the Victorian Alpine National Park, south-eastern Australia. Austral Ecology 26: 670-679.

White, J., Ryan, K., Key, C. and Running, S., 1996. Remote sensing of forest fire severity and vegetation recovery. International Journal of Wildland Fire 6: 125-136.

Wicks, T., Smith, G. and Curran, P., 2002. Polygon-based aggragation of remotely sensed data for regional ecological analysis. International Journal of Applied Earth Observation and Geoinformation 4: 161-173.

Wittenberg, L., Malkinson, D., Beeri, O., Halotzy, A. and Tesler, N., 2007. Spatial and temporal patterns of vegetation recovery following sequences of forest fires in a Mediterranan landscape, Mt. Carmel Israel. Catena 71: 76-83.

Wulder, M. A., White, J. C., Alvarez, F., Han, T., Rogan, J. and Hawkes, B., 2009. Characterizing boreal forest wildfire with multi-temporal Landsat and LIDAR data. Remote Sensing of Environment 113: 1540-1555. 


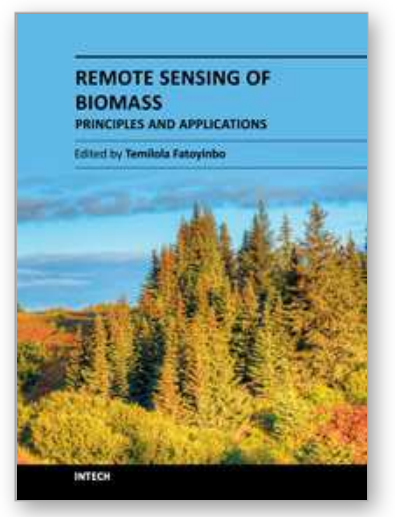

\author{
Remote Sensing of Biomass - Principles and Applications \\ Edited by Dr. Lola Fatoyinbo
}

ISBN 978-953-51-0313-4

Hard cover, 322 pages

Publisher InTech

Published online 28, March, 2012

Published in print edition March, 2012

The accurate measurement of ecosystem biomass is of great importance in scientific, resource management and energy sectors. In particular, biomass is a direct measurement of carbon storage within an ecosystem and of great importance for carbon cycle science and carbon emission mitigation. Remote Sensing is the most accurate tool for global biomass measurements because of the ability to measure large areas. Current biomass estimates are derived primarily from ground-based samples, as compiled and reported in inventories and ecosystem samples. By using remote sensing technologies, we are able to scale up the sample values and supply wall to wall mapping of biomass. Three separate remote sensing technologies are available today to measure ecosystem biomass: passive optical, radar, and lidar. There are many measurement methodologies that range from the application driven to the most technologically cutting-edge. The goal of this book is to address the newest developments in biomass measurements, sensor development, field measurements and modeling. The chapters in this book are separated into five main sections.

\title{
How to reference
}

In order to correctly reference this scholarly work, feel free to copy and paste the following:

Ioannis Gitas, George Mitri, Sander Veraverbeke and Anastasia Polychronaki (2012). Advances in Remote Sensing of Post-Fire Vegetation Recovery Monitoring - A Review, Remote Sensing of Biomass - Principles and Applications, Dr. Lola Fatoyinbo (Ed.), ISBN: 978-953-51-0313-4, InTech, Available from:

http://www.intechopen.com/books/remote-sensing-of-biomass-principles-and-applications/advances-inremote-sensing-of-post-fire-monitoring-a-review

\section{INTECH}

open science | open minds

\author{
InTech Europe \\ University Campus STeP Ri \\ Slavka Krautzeka 83/A \\ 51000 Rijeka, Croatia \\ Phone: +385 (51) 770447 \\ Fax: +385 (51) 686166 \\ www.intechopen.com
}

\author{
InTech China \\ Unit 405, Office Block, Hotel Equatorial Shanghai \\ No.65, Yan An Road (West), Shanghai, 200040, China \\ 中国上海市延安西路65号上海国际贵都大饭店办公楼405单元 \\ Phone: +86-21-62489820 \\ Fax: +86-21-62489821
}


(C) 2012 The Author(s). Licensee IntechOpen. This is an open access article distributed under the terms of the Creative Commons Attribution 3.0 License, which permits unrestricted use, distribution, and reproduction in any medium, provided the original work is properly cited. 